\title{
Graphene fiber: a new material platform for unique applications
}

\author{
Huhu Cheng, Chuangang Hu, Yang Zhao and Liangti Qu
}

Graphene fiber (GF) is of practical importance because it integrates the remarkable properties of individual graphene sheets into useful, macroscopic ensembles that possess the common characteristics of fibers, such as mechanical flexibility for textiles, while maintaining the unique advantages over conventional carbon fibers, such as low cost, light weight, shapeability and ease of functionalization in an in situ or post-synthetic manner for various applications. In this review, we judiciously summarize the significant advances in GFs achieved by our group and others in recent years, including the tunable and controllable preparation of GFs with functionality and their remarkable applications for unconventional devices, such as flexible fiber-type actuators, robots, motors, photovoltaic cells and supercapacitors.

NPG Asia Materials (2014) 6, e113; doi:10.1038/am.2014.48; published online 18 July 2014

\section{INTRODUCTION}

Carbon fibers are widely used in daily life because of their light weight, high mechanical strength and environmental stability. As one of the carbon-based fibers, the emergence of carbon nanotube (CNT) fibers/yarns has attracted tremendous attention as a result of the integration of such properties as high strength and high electrical and thermal conductivities of the individual nanotubes into useful, macroscopic ensembles beyond those of common carbon fibers. ${ }^{1-8}$ However, the limitations that result from the high cost of producing the initial superaligned CNT arrays $s^{5,7,8}$ and the extremely rigorous conditions, such as high temperature $\left(>1000^{\circ} \mathrm{C}\right)^{6}$ and caustic media (for example, fuming sulfuric acid ${ }^{2}$ and chlorosulfonic acid ${ }^{4}$ ), used in their production must be overcome for CNT technology to become widely used.

Graphene, a two-dimensional (2D) monolayer of carbon atoms packed into a honeycomb lattice, is the basic building block for carbon materials of all other dimensionalities. ${ }^{9}$ This material gains immense recognition because of its fascinating properties, such as high electron mobility, ${ }^{10-12}$ high thermal conductivity, ${ }^{13}$ and extraordinary elasticity and stiffness. ${ }^{14}$ Therefore, the development of an effective strategy for well-controlled assembly is crucial for integration of the remarkable properties of individual graphenes into advanced, macroscopic and functional structures for practical applications. The assembly of nanoscale graphene sheets into macroscopic 2D film is generally achieved by simple solution filtration, whereas 3D bulk graphene can be obtained through hydrothermal treatment. ${ }^{15}$ In contrast, it appears to be an extraordinary challenge to directly assemble $2 \mathrm{D}$ microscopic graphene sheets into macroscopic fibers. This observation may be a result of the irregular size and shape of chemically derived graphenes and the movable layer-by-layer stacking of graphenes in contrast to the highly tangled CNT assemblies, which could seriously obstruct the formation of GFs and impair their macroscopic mechanical properties. Nevertheless, the recent success in assembling graphenes into macroscopic fibers has inspired extensive interest in these materials because of the lower cost of GFs compared with CNTs and commercial carbon fibers, and their practical importance for specific applications (http://efie.net/a-new-way-to-make-carbon-fiber/). In addition to the useful complementarity of $2 \mathrm{D}$ films and $3 \mathrm{D}$ bulk graphenes, 1D GFs are especially important for textile-enabled materials and devices, and can also act as the building blocks for forming $2 \mathrm{D}$ and $3 \mathrm{D}$ macroscopic structures for practical applications.

To date, great advances have been made from laboratory studies to the potential industrial applications of GFs. However, a comprehensive review that presents the current status and the perspective of GF research is absent in the literature. In this feature article, we focus our discussion on the tunable and controllable preparation of GFs with functionality and their unique applications for unconventional devices, such as flexible fiber-type actuators, robots, motors, photovoltaic cells and supercapacitors.

\section{PREPARATION OF GFS}

Wet spinning of graphene oxide (GO) liquid crystal phases The finding that soluble, chemically oxidized graphene or GO sheets can form liquid crystals (LCs) in a twist-grain-boundary phase-like model with simultaneous lamellar ordering and long-range helical frustrations has led to the opportunity for continuous spinning of this material into macroscopic GO fibers. ${ }^{16}$ GO LCs with lamellar

Key Laboratory of Cluster Science, Ministry of Education of China, Beijing Key Laboratory of Photoelectronic/Electrophotonic Conversion Materials, School of Chemistry, Beijing Institute of Technology, Beiiing, P.R. China

Correspondence: Professor L Qu, Key Laboratory of Cluster Science, Ministry of Education of China, Beijing Key Laboratory of Photoelectronic/Electrophotonic Conversion Materials, School of Chemistry, Beijing Institute of Technology, 5 South Zhongguancun Street, Haidian District, Beijing 100081, P.R. China.

E-mail: lqu@bit.edu.cn

Received 22 February 2014; revised 4 May 2014; accepted 8 May 2014 
structures allow for the dispersion of GO at high enough concentrations suitable for efficient alignment and effective coagulation. Gao et al. ${ }^{16}$ have fabricated neat GO fibers (Figures 1a-c) by loading the GO dispersions into glass syringes and injecting them into a coagulation bath of a $5 \mathrm{wt} \% \mathrm{NaOH} / \mathrm{methanol}$ solution. Subsequently, the GFs were collected by chemical reduction of GO fibers in hydroiodic acid. This approach of wet-spinning GO LCs opens the pathway to large-scale production of flexible GFs, although their mechanical strength needs further enhancement. Later, the same group prepared porous GFs by spinning the liquid crystalline GO gels into liquid nitrogen. ${ }^{17}$ The porosity gives the graphene aerogel fibers a high surface area, affording a simple and effective way to prepare multifunctional fibers.

Other researchers found that the formulation of LC GO 'inks' enabling the spinning of continuously conducting and robust fibers could have a concentration as low as $0.075 \mathrm{wt} \%$, eliminating the need for relatively concentrated spinning dope dispersions. ${ }^{18}$ Dilute LC GO dispersions have proven to be suitable for fiber spinning using a number of coagulation strategies, including non-solvent precipitation, dispersion destabilization, ionic cross-linking and polyelectrolyte complexation. This low concentration enables a wide range of possibilities, including new formulations of LC GO with different materials (CNTs, metallic-based particles, polymers, etc.) that are not accessible in the case of concentrated dispersions because of their viscosity limitations.

Notably, $\mathrm{Yu}$ et al. ${ }^{19}$ subsequently demonstrated that neat and macroscopic GFs can be fluidly spun from common GO suspensions followed by chemical reduction. The curliness-fold formation mechanism of GO fibers has been proposed. This wet-spinning technique facilitates the multifunctionalization of graphene-based fibers with various organic or inorganic components. Other wetspinning techniques for GO suspensions can be found in reference Chen et al. ${ }^{20}$

Wet-spun GO fibers generally have a relatively low tensile modulus, which is directly related to the intrinsic alignment of the GO layers along the fiber. To solve this problem, Tour and co-workers ${ }^{21}$ further utilized large flake GO ( $22 \mu \mathrm{m}$ average diameter $)$ as the building blocks to assemble fibers by the wet-spinning method. The resulting fibers exhibited a one order of magnitude higher modulus than that of previously reported fibers. Upon spinning from high concentrations (7 wt \%) of viscous dope coupled with an appropriate selection of the coagulation solvent, the obtained fibers spun from large flake GO showed high flexibility with an unconventional 100\% knot efficiency (Figure 1d), which has never before been attained for polymer-based fibers.

By modifying the wet-spinning process, our group developed a coaxial two-capillary spinning strategy for continuously engineered production of morphology-controlled graphene-based hollow fibers. ${ }^{22}$ Figure 2a shows a schematic illustration of the setup for the direct spinning of hollow GO fibers in a coagulation bath of methanol solution (Figure 2b). The concentrated GO suspension has a high viscosity, allowing it to be directly bubbled, thus providing the chance to elaborately tune the morphologic character of the resultant GO fibers. Once replacing the inner fluid with compressed air, the obtained hollow GO fibers display a necklace-like structure where each of the hollow microspheres is connected by the fiber (Figure 2c).

To further control the fiber structure for tailored properties and new applications, Cao et al. ${ }^{23}$ introduced shear stress during wet spinning to produce macroscopic ribbon-like GFs with high flexibility. The graphene ribbons showed high performance in many applications, such as elastic strain sensors, flexible counter electrodes for fiber solar cells and fabric electrodes for supercapacitors.

\section{Dimension-confined hydrothermal strategy}

Hydrothermal treatment of GO will induced spontaneous assembly of graphene sheets in the graphene network via the strong interlayer $\pi-\pi$ stacking between graphenes. ${ }^{15}$ Our group ${ }^{24}$ developed a one-step dimension-confined hydrothermal strategy to fabricate neat GFs from aqueous GO suspensions. A glass pipeline of $0.4 \mathrm{~mm}$ in inner diameter was used as the reactor. An aqueous GO suspension of $8 \mathrm{mg} \mathrm{ml}^{-1}$ was injected into the glass pipeline, and subsequently baked at $230{ }^{\circ} \mathrm{C}$ for $2 \mathrm{~h}$ after sealing the two ends of the pipeline. Finally, a GF matching the pipe geometry was produced. Only $1 \mathrm{ml}$ of GO suspension $\left(8 \mathrm{mg} \mathrm{ml}^{-1}\right)$ will generate a GF $>6-\mathrm{m}$ long. The fiber diameter (typically $5-200 \mu \mathrm{m}$ ) and length (typically several meters) can be easily controlled by either using a pipeline with a predesigned length and inner diameter or adjusting the initial GO concentration. As a result of the dense stacking of graphene layers induced by the hydrothermal process, the assembled GFs have a relatively high strength of up to $180 \mathrm{MPa}$.
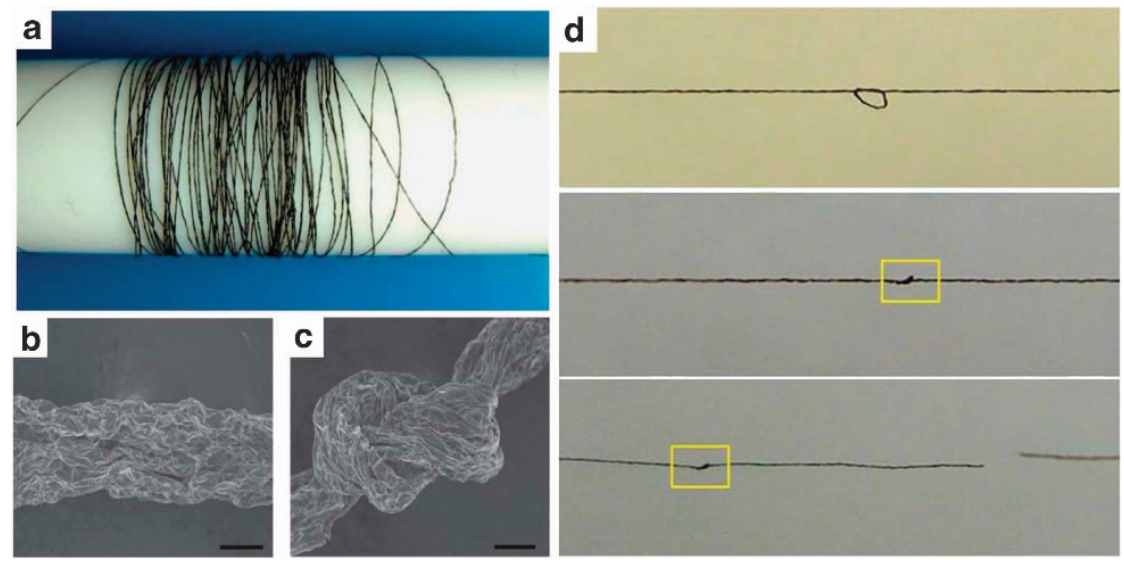

Figure 1 Macroscopic neat graphene oxide (GO) fibers. (a) Four-meter-long GO fiber wound on a Teflon drum (diameter, $2 \mathrm{~cm}$ ). (b) Scanning electron microscope (SEM) image of graphene fiber (GF) and (c) the tightened knot. Scale bars, $50 \mu \mathrm{m}$. Reproduced with permission. Copyright 2011 from reference. (http://efie.net/a-new-way-to-make-carbon-fiber/). (d) A tied large flake GO (LFGO) fiber with continuous pull until it breaks at a point far from the knot. Reproduced with permission. Copyright 2013 from Xiang et al. ${ }^{21}$ 

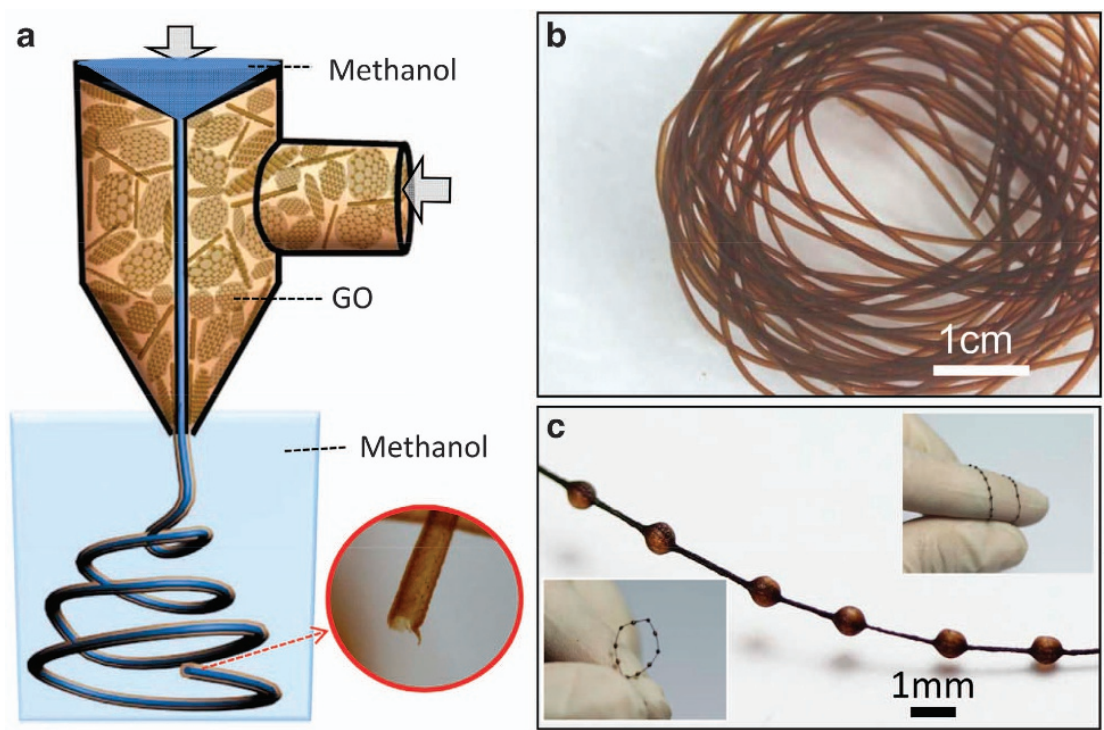

Figure 2 (a) Schematic illustration of the setup using a dual-capillary spinneret to directly spin hollow graphene oxide fibers (hGOFs). (b) Photos of the spun hGOFs in methanol solution and (c) photos of the typical necklace-like hGOFs. Reproduced with permission. Copyright 2013 from Zhao et al. ${ }^{22}$

Based on the hydrothermal method, a dually geometric confinement approach for preparing meter-long hollow GFs (hGFs) with tunable diameters has been further developed. ${ }^{25}$ For example, one removable wire-type template (for example, $\mathrm{Cu}$ wire) was placed within the glass pipeline, which was thermally treated to induce the aggregation of hydrothermally RGO along the wire. Finally, meters of hGFs with diameters depending on the supporting wire template were collected. Interestingly, two-, three- and four-channel hGFs can be readily fabricated by utilizing two-, three- and four-ply $\mathrm{Cu}$ wires, respectively.

\section{Chemical vapor deposition (CVD)-assisted process}

Zhu and co-workers ${ }^{26}$ developed a direct drawing approach to fabricate GFs from CVD grown graphene films. Graphene films were first transferred from the growth substrate to an organic solvent (for example, ethanol) and were then drawn out from the solvent using tweezers to form a fiber-like structure upon the evaporation of the ethanol. The surface tension and the evaporation rate of the solvent have important roles in the formation of porous GF structures. Although the formed fibers have high electrical conductivity $\left(\sim 1000 \mathrm{~S} \mathrm{~m}^{-1}\right)$, this method struggles to continuously produce uniform GFs with desired lengths and strength on a large scale.

The same group utilized atmospheric CVD to directly grow graphene onto $\mathrm{Cu}$ meshes, a network of $\mathrm{Cu}$ wires. ${ }^{27}$ After removing the $\mathrm{Cu}$ wires with an $\mathrm{FeCl}_{3} / \mathrm{HCl}$ aqueous solution, a network configuration of the hGFs was achieved, which is called graphenebased woven fabric (GWF). Graphene-based woven fabrics could be further transferred onto polydimethylsiloxane (PDMS) substrates to produce graphene-based woven fabric/PDMS hybrid films for various applications.

\section{Spontaneous reduction and assembly of GO}

As we have discussed, hGFs can be prepared by CVD growth of graphene along $\mathrm{Cu}$ wires or meshes. In fact, even without the CVD process, hGFs can be spontaneously formed on $\mathrm{Cu}$ wires based on a facile and efficient substrate-assisted reduction and assembly of GO method (Figure 3a). ${ }^{28}$ In this approach, the active metal substrates are
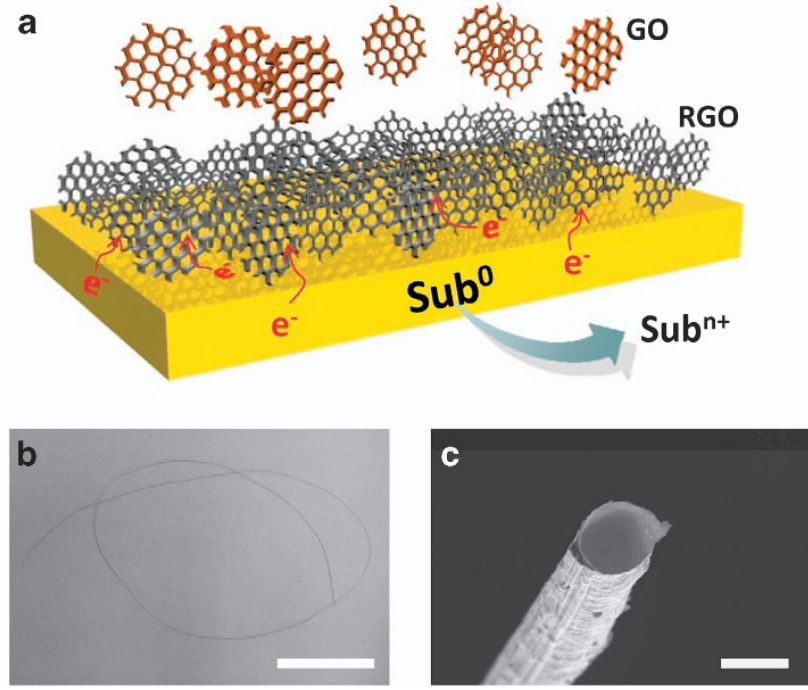

Figure 3 (a) Substrate-assisted reduction and assembly of graphene oxide (SARA-GO) process on active metal substrates. The active metal substrates $\left(\mathrm{Sub}^{0}\right)$ are oxidized to $\mathrm{Sub}^{\mathrm{n}+}$ ions with the loss of an electron $\left(\mathrm{e}^{-}\right)$, whereas the GO is reduced by accepting an electron. (b, c) Photograph and scanning electron microscope (SEM) image of the hollow graphene fiber (hGF) produced by SARA-GO on a Cu wire. Scale bars: b, $1 \mathrm{~cm}$; c, $50 \mu \mathrm{m}$. Reproduced with permission. Copyright 2013 from Hu et al. ${ }^{28}$

oxidized to metal ions with the loss of electrons, while the GO is reduced by accepting electrons. The electron transport through the metal to the GO allows for the 3D assembly of reduced GO (RGO). This method also allows for the reduction of GO and its wellorganized assembly on arbitrary conductive surfaces, such as active metals $\mathrm{Zn}, \mathrm{Fe}$ and $\mathrm{Cu}$; inert metals $\mathrm{Ag}$, Pt and $\mathrm{Au}$; semiconducting $\mathrm{Si}$ wafers; nonmetallic carbon-based films; and even indium-tin oxide glass, without any additional reducing agents. In the case of $\mathrm{Cu}$ wire as the active substrate, the hGFs can be produced by substrate-assisted reduction and assembly of GO as presented in Figures $3 \mathrm{~b}$ and c. This method is favorable for the formation of the structure-defined assembly of graphenes. 
From aligned CNT sheets to graphene yarn

Graphene nanoribbons can be derived from $\mathrm{CNTs}^{29-31}$ that already possess the high aspect ratio. Based on this concept, Baughman et al. ${ }^{32}$ fabricated graphene nanoribbon yarns by chemically unzipping sheets of highly aligned CNTs and densifying them during the drying process. In this case, the original multiwalled CNT sheets were first layered onto a polytetrafluoroethylene frame that allowed for the unzipped GO nanoribbon (GONR) sheet to collapse onto a GONR yarn when removed from the liquid-phase solution. Specifically, multiwalled CNT layers wrapped vertically around a polytetrafluoroethylene framework immersed in solution (first an oxidizing bath, followed by a rinsing solution) render GONR sheets. After drying at room temperature, the GONR yarn shrinks from $\sim 1 \mathrm{~mm}$ to $\sim 25 \mu \mathrm{m}$ in diameter. A combination of different reduction treatments can tune the presence/absence of superficial chemical groups useful for improving their mechanical, electrical and electrochemical properties. In fact, GONRs and chemically reduced graphene nanoribbons can also be dispersed at high concentrations in chlorosulfonic acid to form anisotropic LC phases for wet spinning of GFs. ${ }^{33}$

\section{Other methods}

Although the aforementioned methods provide a considerable number of options to controllably prepare different types of GFs for diverse purposes, other methods have been reported. Kim and co-workers reported an electrophoretic assembly method for the fabrication of reduced GONR fibers. ${ }^{34}$ A graphitic tip as a positive electrode was immersed into the chemically reduced GONR colloidal solution in which the counter electrode was embedded. A constant voltage ranging from 1 to $2 \mathrm{~V}$ was applied between the electrodes during the withdrawal process for the graphitic tip $\left(0.1 \mathrm{~mm} \mathrm{~min}^{-1}\right)$. Similar to the direct drawing method mentioned above, this approach also has relatively low production efficiency unsuitable for large-scale processes. Alternatively, $\mathrm{Xu}$ et $a l^{35}$ reported a facile solution selfassembly strategy to fabricate neat GO fibers from aqueous GO suspensions at the liquid/air interface based on repulsive electrostatic forces, attractive van der Waals forces and $\pi-\pi$ stacking. During the assembly process and ultrasonic cleaning, the morphology interestingly varied from the source graphite powder to GO sheets, then to GO fibers and finally to neat GO fiber films. This method provides a simple approach toward the scalable preparation of random GFs with a small diameter of $1-2 \mu \mathrm{m}$ and a length of approximately hundreds of micrometers, although the formation mechanism may need further study.

Thus, various methods are available for the fabrication of different types of GFs with specific features (Table 1), which provides abundant material platforms that are unique for different applications, as further demonstrated below.

\section{FUNCTIONAL COMPOSITES}

\section{Intercalation of functional components}

Integration of functional components into GFs will facilitate important applications, such as stimulus-responsive and electronic textiles. The GFs provide a platform for in situ and post-synthetic incorporation of various materials with unique properties into the fiber bodies for weavable, multifunctional applications. ${ }^{36,37}$ For example, magnetic GFs have been fabricated by in situ introduction of $\mathrm{Fe}_{3} \mathrm{O}_{4}$ nanoparticles into the fibers. ${ }^{24}$ The $\mathrm{Fe}_{3} \mathrm{O}_{4}$-incorporated GFs with good mechanical flexibility exhibit a sensitive magnetic response. As demonstrated in Figure $4 \mathrm{a}$, the magnetized GFs can iteratively bend to the magnet from its initial upright position.
As an example of post-synthetic functionalization, $\mathrm{TiO}_{2}$ nanoparticles have been intercalated into the framework of graphene sheets by soaking the wet as-prepared GFs in a commercial $\mathrm{TiO}_{2}$ aqueous suspension with slight stirring. ${ }^{24}$ After drying and annealing, the $\mathrm{TiO}_{2}$-GFs exhibit a fast photocurrent response with good repeatability (Figure 4b). The strong photocurrent response indicates a direct electron/hole injection between the $\mathrm{TiO}_{2}$ particles and graphene sheets through photoexcitation of $\mathrm{TiO}_{2}$, suggesting the significant applications in optoelectronic systems, such as photodetectors, photocatalysts and photovoltaic cells. However, selective functionalization can be achieved in a site-specific outer-wall, inner-wall, outer/inner-wall and within-wall fashion on hGFs. ${ }^{25}$

To improve the electrical conductivity of GFs, Gao et al..$^{38}$ prepared graphene-metal hybrid fibers by wet spinning of GO LCs mixed with commercial Ag nanowires, followed by chemical reduction (Figure 4c). The Ag-doped GFs exhibited a record high electrical conductivity up to $9.3 \times 10^{4} \mathrm{~S} \mathrm{~m}^{-1}$ and a high current capacity of $7.1 \times 10^{3} \mathrm{~A} \mathrm{~cm}^{-2}$. The combination of high conductivity and high mechanical strength and flexibility of doped GFs renders them ideal stretchable conductors to be applied in soft circuits.

Aside from their high electrical conductivity, excellent mechanical properties are also highly desired for GFs. Generally, a large aspect ratio of the building blocks and better alignment in the fiber axis are the main factors. It was also found that the introduction of divalent ionic cross-linking between graphene sheets could further enhance their mechanical strength. ${ }^{39}$ As an example, a record tensile strength of up to $0.5 \mathrm{GPa}$ has been achieved among neat graphene materials (Figure 4d).

To achieve strong GFs, Gao et al. ${ }^{40}$ further produced bio-mimic continuous fibers with 'brick-and-mortar' structures of alternating graphene sheets and hyperbranched polyglycerol binders via wetspinning assembly technology. The formation of strong and adaptive hydrogen bonding networks between GO and hyperbranched polyglycerol contributes to their super properties. The optimized results ${ }^{41}$ showed that the biomimetic composites achieved record high tensile strength $(0.65 \mathrm{GPa})$ and toughness $\left(18 \mathrm{MJ} \mathrm{m}^{-3}\right)$ among nacre mimics. In addition, the composites exhibited high electrical conductivity $\left(5261 \mathrm{~S} \mathrm{~m}^{-1}\right)$ comparable to that of neat graphene papers. Meanwhile, it was discovered that polymer-grafted GO fibers show remarkable chemical resistance, even to $98 \%$ sulfuric acid, because of their compact layered structures and strong interlayer interactions. ${ }^{42}$ As a result of the covalent and uniform immobilization of polymer chains on the surface of individual GO sheets, local phase separation is fundamentally avoided and strong interfacial interactions are simultaneously introduced. Polymer chains were then covalently bonded to the nanoplatelets through in situ free radical polymerization of vinyl monomers (for example, glycidyl methacrylate).

\section{All-carbon composites}

Graphenes are typical 2D nanostructures with unique properties, whereas CNTs are important 1D nanomaterials. Thus, the hybrid of these two materials could possess interesting properties. Li et al. ${ }^{43}$ achieved CNT and graphene hybrid multiple-thread yarns, consisting of tens or hundreds of single-thread CNTs, from a chemical vapor gas flowing reaction followed by post-treatment processing. A thick CNT stick-like assembly was first fabricated by CVD processing with a horizontal reactor. CNT and graphene hybrid multiple-thread yarns were then drawn from the stretched, thick stick-like CNT assembly. The as-obtained CNT and graphene hybrid multiple-thread yarns can be twisted to form one fiber. The mechanical measurement of the 
Table 1 Brief summary of the typical features of GFs fabricated by different methods

\begin{tabular}{|c|c|c|c|c|c|c|c|}
\hline Preparation method of GFs & Flexibility & $\begin{array}{c}\text { Diameter } \\
(\mu \mathrm{m})\end{array}$ & Strength (MPa) & $\begin{array}{l}\text { Young's modulus } \\
\text { (GPa) }\end{array}$ & $\begin{array}{l}\text { Conductivity } \\
\left(\mathrm{Scm}^{-1}\right)\end{array}$ & Remarks & Ref. \\
\hline \multirow[t]{3}{*}{ Spinning of GO } & High & $50-100$ & 140 & 5.4 & $\sim 250^{\mathrm{a}}$ & Scalable & $\mathrm{Xu}$ and $\mathrm{Gao}^{16}$ \\
\hline & & $40-150$ & 85 to 192 & 2.8 to 9.8 & $68-230^{a}$ & Scalable & Chen et al. ${ }^{20}$ \\
\hline & & $<100$ & $442 \pm 18$ & $22.6 \pm 1.9$ & $28.44^{b}$ & Scalable & Jalili et al. ${ }^{18}$ \\
\hline CVD assistant process & Low & $20-50$ & & & $\sim 10$ & & Li et al. ${ }^{26}$ \\
\hline $\begin{array}{l}\text { Spontaneous reduction and } \\
\text { assembly of GO }\end{array}$ & Low & $15-100$ & & & $\sim 10$ & Morphology predefined & Hu et $a .^{28}$ \\
\hline $\begin{array}{l}\text { Aligned CNT sheets to } \\
\text { graphene yarn }\end{array}$ & High & $\sim 100$ & & & & $\begin{array}{l}\text { Up to } 100 \mathrm{Scm}^{2} \mathrm{~g}^{-1} \text { after } \\
\text { annealing }\end{array}$ & $\begin{array}{l}\text { Carretero-Gonzalez } \\
\text { et al. }\end{array}$ \\
\hline Electrophoretic self-assembly & & $20-40$ & & & & & Jang et al. ${ }^{34}$ \\
\hline
\end{tabular}

Abbreviations: CNT, carbon nanotube; CVD, chemical vapor deposition; GF, graphene fiber; GO, graphene oxide; GONR, GO nanoribbon.

aAfter chemical reduction.

${ }^{\mathrm{b}}$ After annealing at high temperature.
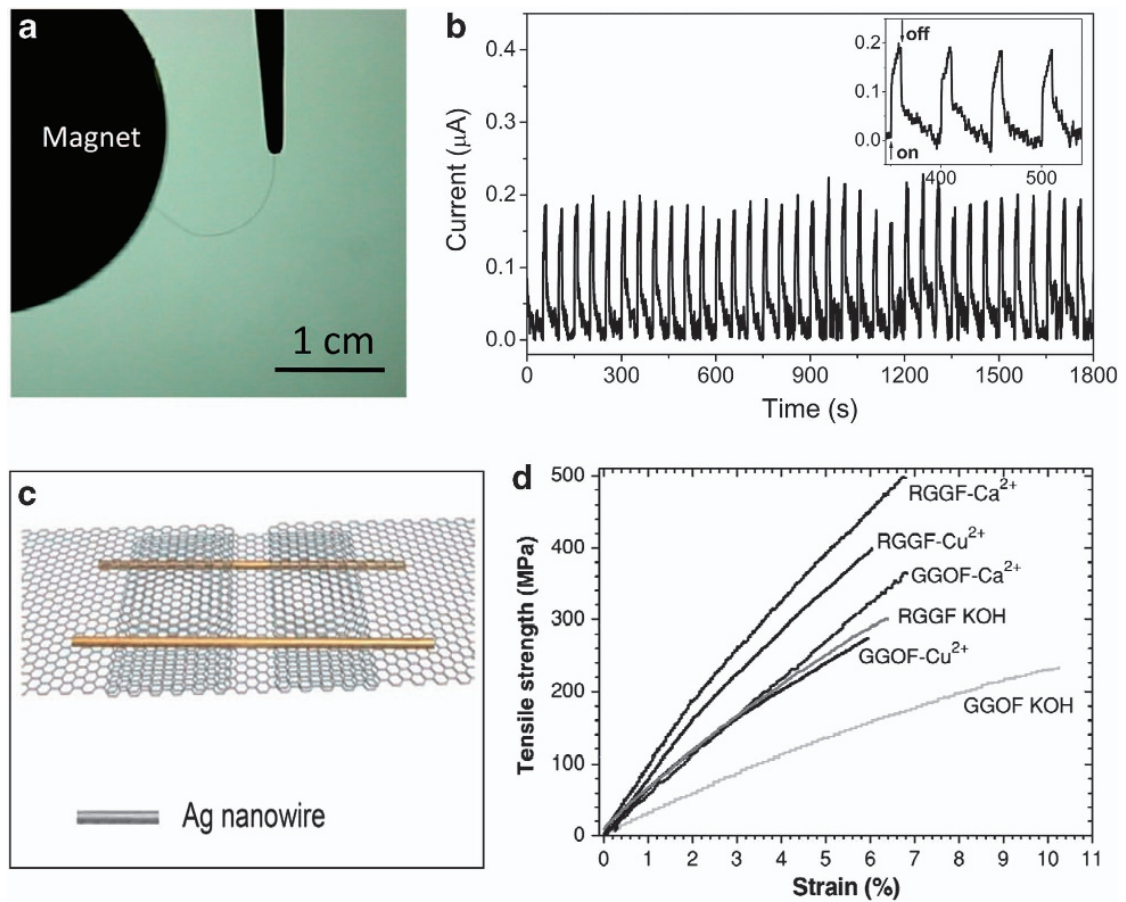

Figure 4 (a) Magnetic $\mathrm{Fe}_{3} \mathrm{O}_{4}$ /graphene fiber (GF) attracted to the magnet. (b) A typical photocurrent response for a $\mathrm{TiO}_{2}$ immobilized GF upon exposure to on/off light at room temperature. The inset shows an enlarged view for a small portion of the photocurrent response curve. Reproduced with permission. Copyright 2012 from Dong et al. ${ }^{24}$ (c) Possible complex mechanism by Ag nanowires with graphene sheets. Reproduced with permission. Copyright 2013 from Xu et al. ${ }^{38}$ (d) Mechanical measurements under tension for GFs cross-linked by different divalent ions. Reproduced with permission. Copyright 2013 from Xu et al. ${ }^{39}$

twisted yarn resulted in a strength of $300 \mathrm{MPa}$ and the electrical conductivity was found to be $10^{5} \mathrm{~S} \mathrm{~m}^{-1}$.

Another method is to directly grow the CNTs from the GF. Cheng et al. ${ }^{44}$ prepared GFs with intercalated $\mathrm{Fe}_{3} \mathrm{O}_{4}$ nanoparticles by a hydrothermal process, which were then treated through the CVD process for growth of CNTs along the GFs. Although the hybrid fibers have relatively low mechanical strength, a flexible textile of CNT/GFs has been prepared and used as an electrode for the construction of flexible supercapacitors.

In contrast to the graphene/CNT fibers, a unique all-graphene core-sheath fiber has been fabricated in which a core of GF is covered with a sheath of 3D porous network-like graphene framework, ${ }^{45}$ named GF@3D-G. 3D graphene structures exhibit notable features, including highly exposed surface areas, high electrical conductivity 
a
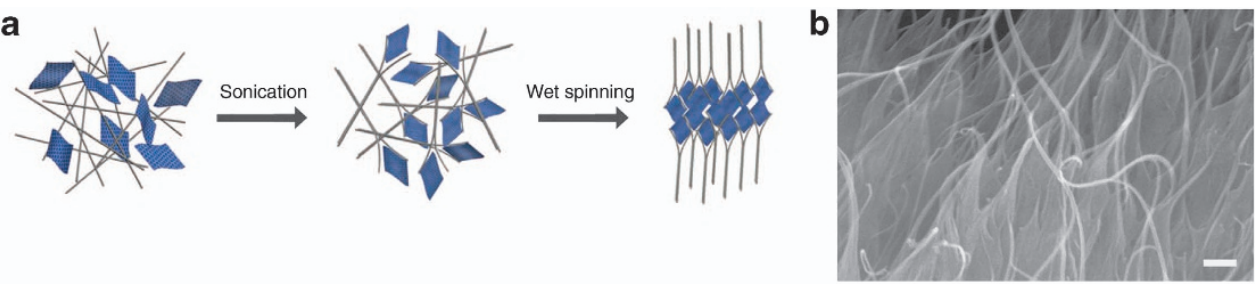

c
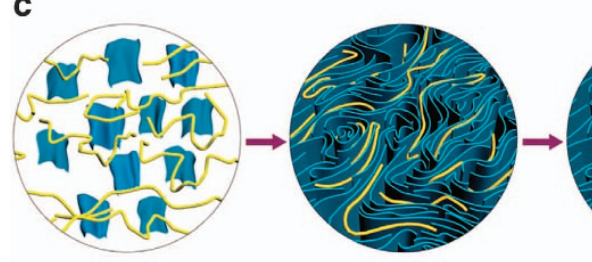

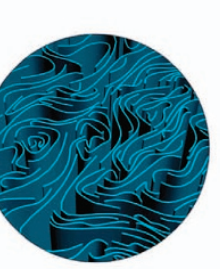

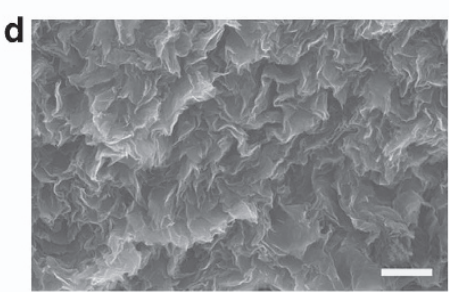

Figure 5 Preparation and structure of polymer composite fibers. (a) Schematic diagram showing the formation of the oriented interconnected network of reduced graphene oxide (RGO) flakes (curved rectangles) and single-walled carbon nanotube (SWNT) bundles (grey lines) as a result of sonication and subsequent wet-spinning. (b) Scanning electron microscope (SEM) image of the cross-sectional area of a RGO/SWNT/poly(vinyl alcohol) (PVA) fiber, which clearly shows the co-assembly of RGO flakes and SWNTs. Scale bar, $100 \mathrm{~nm}$. (c) Schematic illustration of the structural evolution between coagulation-spun RGO gel (left), polymer composite (middle) and polymer-free fibers (right). Yellow lines and blue curved rectangles represent PVA chains and RGO flakes, respectively. (d) SEM image of the cross-sectional area of a polymer-free RGO fiber, which clearly shows the wrinkled RGO flakes. Scale bar, 500 nm. Reproduced with permission. Copyright 2012 from Shin et al. ${ }^{46}$

and good chemical stability. Herein, the high electronic conduction of the core GF and the highly exposed surface area of the 3D graphene network are combined within the GF@3D-G, thus offering the great advantages as flexible electrodes for efficient fiber-based devices.

\section{Polymer composites}

Previous work has shown that the toughness of the CNT-reinforced polymer fibers exceeds that of previously known materials. ${ }^{1}$ Kim et al. $^{46}$ showed further increased toughness results from the combination of CNTs with RGO flakes in solution-spun polymer fibers. The process for fabricating hybrid fibers based on RGO flakes and single-walled carbon nanotubes (SWNTs) is shown in Figure 5a. Uniform dispersions of various relative amounts of RGOs and SWNTs in aqueous solutions of sodium dodecyl benzene sulfonate were prepared. The dispersions were then injected into the flow stream of an aqueous solution of $5 \mathrm{wt} \%$ poly(vinyl alcohol) (PVA) dissolved in deionized water, which resulted in coagulation and formation of RGO/SWNT/PVA hybrid fibers. After washing in deionized water, drying and subsequently treating with methanol to increase PVA crystallinity, mechanically robust hybrid fibers consisting of RGO/ SWNT imbedded in a PVA matrix were obtained (Figure 5b). Figure $5 c$ schematically illustrates the transformation of as-spun gel fibers into solid RGO/PVA fibers and then polymer-free fibers of wrinkled RGO flakes. The polymer-free fibers were mechanically stable, indicating the formation of an interconnected network of RGO flakes (Figure 5d). The polymer composite fibers have a gravimetric toughness of $1000 \mathrm{Jg}^{-1}$, far exceeding that of spider dragline silk $\left(165 \mathrm{Jg}^{-1}\right)$ and Kevlar $\left(78 \mathrm{Jg}^{-1}\right)$. This toughness enhancement is consistent with the observed formation of an interconnected network of partially aligned RGO flakes and CNTs during solution spinning, which act to deflect cracks and allow energy-consuming polymer deformation. The hybrid fibers were sewable and weavable, and could be shaped into high-modulus helical springs.

In fact, graphene as a filler has been widely studied for polymer composites. The addition of a low fraction of graphene nanosheets could result in a significant improvement in the mechanical strength and electrical properties of the composites. Graphene nanosheets provide good integration with a polymer matrix because of its planar structure with large interfacial area for $\pi-\pi$ stacking with the polymer matrix. In addition, the carboxyl and hydroxyl functional groups on the basal planes and edges of the starting GO nanosheets may act as linkers between the graphene and the polymer. For example, the graphene nanoribbon/carbon composite nanofiber yarns were prepared by electrospinning from poly(acrylonitrile) containing GONRs, followed by successive twisting and carbonization. ${ }^{47}$ The electrospinning process can exert directional shear force coupling with the external electric field to the flow of the spinning solution. During electrospinning, the well-dispersed GONRs were highly oriented along the fiber axis in an electrified thin liquid jet. The addition of GONRs at a low weight fraction significantly improved the mechanical properties of the composite nanofiber yarns. Typical graphene-polymer nanofibers fabricated by the electrospinning method have been reported on the basis of PVA, ${ }^{48,49}$ poly(vinyl acetate $)^{50}$ and poly-acrylic acid. ${ }^{51}$

\section{UNIQUE APPLICATIONS}

\section{New type of functional fibers}

The pliability of GFs facilitates their weaving into a variety of macroscopic objects. For example, a textile structure of GFs with self-supported stability can be achieved (Figure 6a), ${ }^{24}$ which displays great potential for electronic textile applications in view of the good conductivity of GFs. The flexible and mechanically stable GFs can also be shaped into specific geometries on demand, as exemplified in Figure $6 \mathrm{~b}$ for a GF spring with stretchable and compressible character. ${ }^{24}$ In addition, the GFs can be expediently integrated into flexible, transparent, conductive composite films. Figure $6 \mathrm{c}$ shows a GF network embedded into a PDMS matrix with tolerance to deformation. ${ }^{24}$ Similarly, the GF can also be knitted in cotton textiles (Figure 6d). ${ }^{16}$ Others, such as the specifically defined configurations of four-way pipe (Figures $6 \mathrm{e}$ and $\mathrm{f})^{28}$ with tunable length and diameter, can also be readily prepared.

Ag-doped GFs have high electrical conductivity with high flexibility that makes them attractive for flexible conductors. Gao et al. ${ }^{38}$ prepared a stretchable array of graphene-Ag fibers (Figure 7a) on 

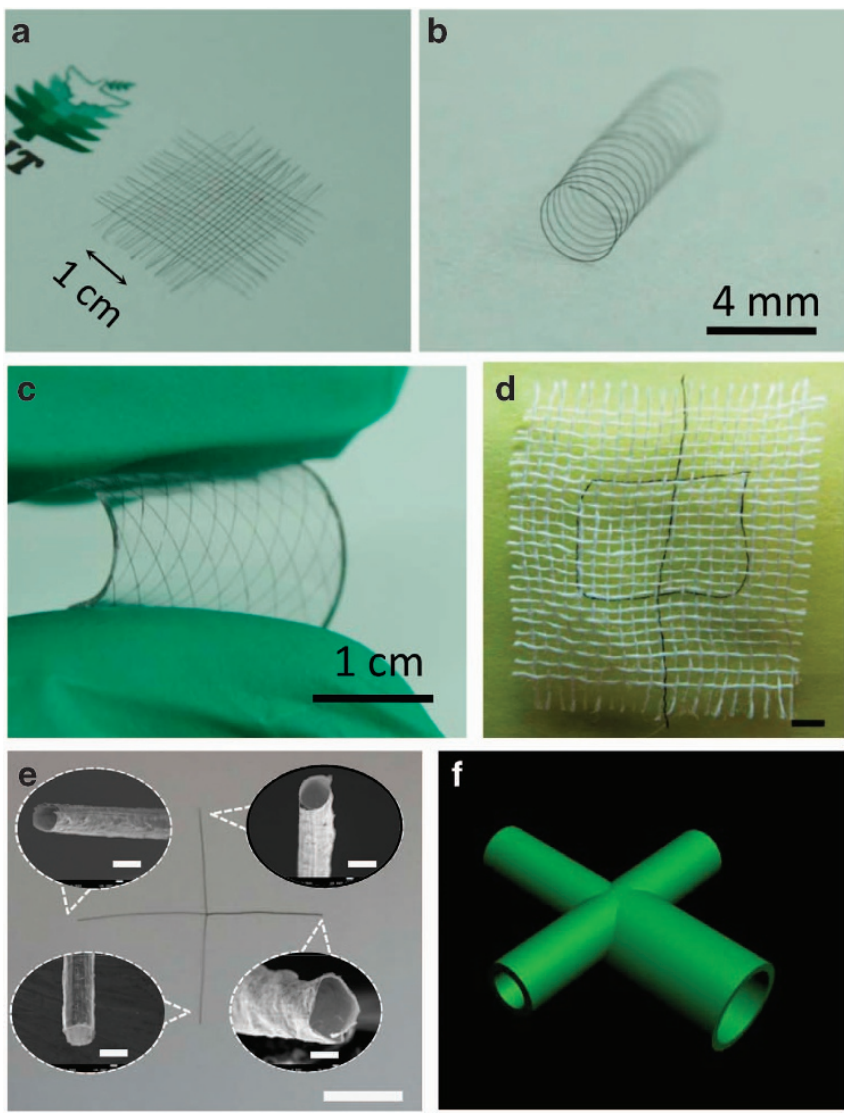

Figure 6 (a) Photographs of the hand-knitted textile of graphene fibers (GFs). (b) The spring of the GF. (c) Photograph of a GF network embedded in a polydimethylsiloxane (PDMS) matrix. Reproduced with permission. Copyright 2012 from Dong et al.24 (d) A GF pattern knitted in the cotton network (white) using two GFs (black). Scale bars, $2 \mathrm{~mm}$. Reproduced with permission. Copyright 2011 from Xu et al. ${ }^{16}$ (e) Photograph of a four-way tube with the corresponding SEM images of the nozzles, and (f) the corresponding schematic diagram. Scale bars: e, $1 \mathrm{~cm}$; e inset, $100 \mu \mathrm{m}$. Reproduced with permission. Copyright 2013 from Hu et al. ${ }^{28}$

PDMS substrates. The Ag-GFs were fixed to $\mathrm{Al}$ foils by silver glue on the $150 \%$ prestrained PDMS substrate. Under compression in the fiber axis during the strain relaxation, the Ag-GFs buckled to become curved threads without breakage because of their good flexibility (Figure 7a). The stretchable conductors can be safely stretched below $150 \%$ strain under electrical loading. As shown in Figure $7 \mathrm{~b}$, the constructed circuit kept working after 150\% stretching, as indicated by the light emitting diode chips.

\section{GF actuators}

Smart materials and structures with fast, reversible and controllable shape changes in response to environmental stimuli have attracted tremendous attention because of their remarkable potential in applications ranging from robots and sensors to memory chips. Graphene-based structures exhibit many exciting properties that could benefit such actuation systems, ${ }^{52-57}$ for example, electrochemical actuators based on unimorph and bilayer graphene films and 3D graphene skeletons. ${ }^{58-61}$ GFs possess the mechanical flexibility required for textiles, yet are unique in that they are lightweight and easy to functionalize in comparison with conventional carbon fibers.
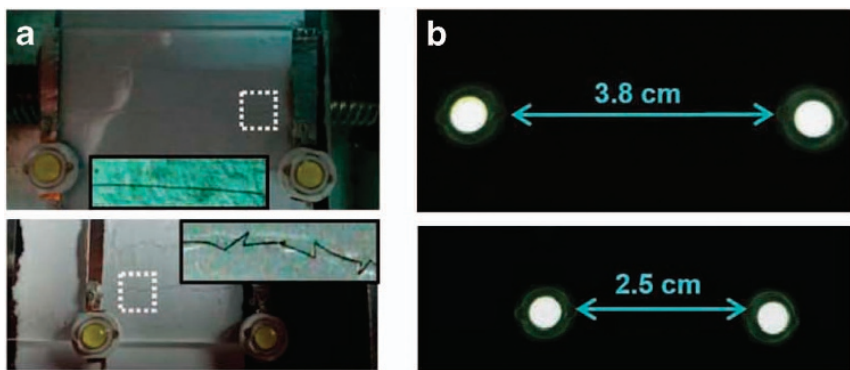

Figure 7 (a) The stretchable array constructed by Ag-graphene fibers (GFs) in the stretched (top) and relaxed state (bottom). The inserted images are magnified photos of the Ag-GFs in the respective areas indicated by the rectangles. (b) The working states of Ag-GF-based stretchable circuit in the stretched (top) and relaxed state (bottom). Reproduced with permission. Copyright 2013 from $\mathrm{Xu}$ et al. ${ }^{38}$

These remarkable features provide graphene with prominent advantages for the development of unconventional, flexible, stimulus-responsive structures, especially in fiber form. A novel electrochemical fiber actuator based on GF/polypyrrole bilayer structures has been recently demonstrated. ${ }^{62}$ The fiber actuator was found to have a high actuation activity and durability comparable to other graphenebased actuators. Multi-armed tweezers and net actuators have been fabricated, which exhibit great promise in different fields including biological studies and microfabrication.

Our group ${ }^{63}$ rationally designed and fabricated graphene/GO (G/ GO) asymmetric fibers as a result of the positioned laser reduction of freshly spun GO fibers (Figures 8a and b). Remarkably, the G/GO fibers display complex, well-confined and predetermined motion and deformation once exposed to moisture. The excellent humidityresponsive bending/unbending ability of the G/GO fibers enabled a new type of microfiber walking device. The G/GO fiber walker can travel in a narrow slit. As shown in Figure 8c, the G/GO fiber walker was placed between two glass slides with a gap of approximately $1 \mathrm{~mm}$, which is also supported by a ratchet substrate. Initially, the G/ GO fiber walker stands under ambient conditions (Figure 8d). Once the relative humidity increases, its back end shifts because of the increased bending (Figure 8e). During the stretching process induced by the removal of moisture, the front end of the G/GO fiber walker slides forward, while the back tip is fixed by the indentations on the rough surface of the substrate (Figure $8 \mathrm{f}$ ). With the alternation of the relative humidity, the device walks unidirectionally step-by-step with a moving rate of $4 \mathrm{~mm}$ per cycle. In principle, the moving speed and motion of this proof-of-concept prototype for the walking robot could be readily improved by regulating the frequency of the humidity alternation and also the device length.

\section{GF motors}

GFs typically possess in-line assembled graphene sheets that are induced during the fiber formation process. By reconstructing the intrinsic configuration of graphenes within the fiber body, a new type of moisture-driven rotational motors ${ }^{64}$ can be achieved by rotary processing of the freshly spun GO fiber hydrogel (Figures $9 a-c)$. As a result of the oxygen-rich functional groups of GO, the relatively fast and reversible expansion/contraction of GO layers will take place through the adsorption and desorption of water molecules. Therefore, the formed helical geometry of GO fibers will enable the reversible torsional rotation under the alternation of humidity (Figure 9d). The maximum torsional rotation rate can reach 5190 rotations min $^{-1}$ $\left(543 \mathrm{rad} \mathrm{s}^{-1}\right)$ and the entire rotation process contains 327 full turns. 


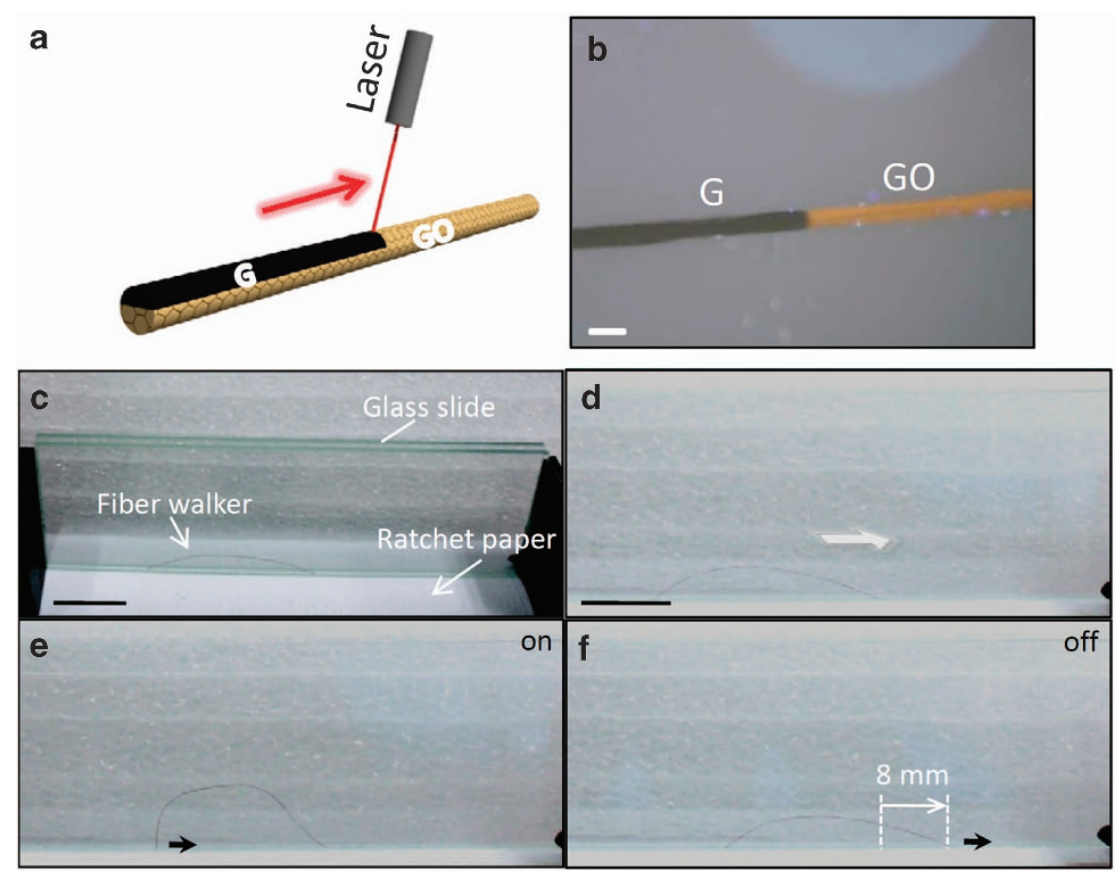

Figure 8 (a) Representation of positioned laser reduction on one side of a graphene oxide (GO) fiber. The black region corresponds to the laser-induced G region along the brown GO fiber. (b) Photomicrograph of the top surface of the asymmetric graphene/GO (G/GO) fiber. Scale bar, $50 \mu \mathrm{m}$. (c) Photograph of the G/GO fiber walker within the narrow slit between two glass slides on the ratchet paper. (d) The initial state of the G/GO fiber walker, which is manually bent slightly in advance to provide a standing pose. (e, f) A series of photographs of the G/GO fiber walker crawling on the ratchet paper in response to the 'on' and 'off' states of applied moisture. The G side of G/GO fiber is placed downward. Relative humidity (RH; 'on' state) $=80 \%, \mathrm{RH}$ ('off' state) $=25 \%$. Scale bars in (c, d), $1 \mathrm{~cm}$. Reproduced with permission. Copyright 2013 from Cheng et al. ${ }^{63}$

The torsional rotation of twisted GF (TGF) enabled the fabrication of a new type of humidity switch. As shown in Figure 9e, when the environment humidity increases, the TGF will drive the paddle rotation, which triggers the switch and turns on the light emitting diode (Figure 9e, inset). Upon removal of the moisture, the TGF will pull the paddle to its original position and release the press on the aluminum pad, hence switching off the light.

These unique TGFs that are sensitive in response to moisture also provide the opportunity to develop a humidity-triggered electric generator, which will produce power using mechanical work induced by the variation of ambient moisture. As shown in Figure 9f, when the entire device was exposed to an environment with varying humidity, the TGF could drive the magnet in a reversible rotation, thus leading to the generation of electricity in the $\mathrm{Cu}$ coils. This generator produced an open-circuit voltage of up to $1 \mathrm{mV}$ (Figure 9g), and a short-circuit current of up to $40 \mu \mathrm{A}$ (Figure 9h) despite the absence of device optimization.

Micro-motors propelled by self-generated forces have gained substantial interest in the scientific community. The ability of the transport and delivery of cargo in various environments is of great importance. ${ }^{65-67}$ Based on the site-specific functionalized hGF, a selfpowered graphene micromachine can be designed that will move in aqueous medium. ${ }^{25}$ As illustrated in Figure 10a, the inner-wall modified hGF with $\mathrm{Pt}$ nanoparticles is ideal as a transporting motor. Similar to the tubular microjet, the $\mathrm{O}_{2}$ bubbles from $\mathrm{H}_{2} \mathrm{O}_{2}$ decomposition were thrust out at one open end, causing a fast and directional movement, whereas the Pt nanoparticles were the catalyst for the decomposition of $\mathrm{H}_{2} \mathrm{O}_{2}$ to produce $\mathrm{O}_{2}$. Once sealed in one end of the hGF, the ejected $\mathrm{O}_{2}$ bubbles from the other end will drive the hGF in the opposite direction. The fast motion of an hGF micromotor ( $6 \mathrm{~mm}$ in length and $100 \mu \mathrm{m}$ in diameter) in $20 \mathrm{wt} \%$
$\mathrm{H}_{2} \mathrm{O}_{2}$ aqueous solution is recorded in Figures 10b-h. As seen in the figure, a large number of gas bubbles are jetted out from the hGF tail, which pushes the hGF a distance of approximately $24 \mathrm{~cm}$ within $6 \mathrm{~s}$. This proof-of-concept hGF motor demonstrated the potential of sitespecific modification of hGFs for advanced self-powered micromotors for various applications.

\section{Dye-sensitized photovoltaic wires}

The combined remarkable mechanical and electrical properties of GFs ensure them to be a promising new family of electrode materials. Peng et al. ${ }^{68}$ developed novel wire-shaped photovoltaic devices based on graphene/Pt composite fibers as counter electrodes, and $\mathrm{Ti}$ wire impregnated with $\mathrm{TiO}_{2}$ nanotubes as working electrodes (Figure 11). The high flexibility, mechanical strength and electrical conductivity of GFs resulted in a certified maximum energy conversion efficiency of $8.45 \%$, which is much higher than that of other wire-shaped photovoltaic devices. These photovoltaic wires have the potential to be easily woven into clothes, packages and other portable devices to serve as self-powered electric generators by conventional textile technology.

\section{GF-based supercapacitors}

Graphene materials are also attractive for applications in electrochemical capacitors. ${ }^{69,70}$ Conventional supercapacitors are heavy and bulky, targeted for applications in electric or hybrid vehicles and auxiliary power sources. However, the development of high-efficiency miniaturized supercapacitor devices that are compatible with flexible and wearable electronics is lacking. Combining the high electrical conductivity of GFs with the highly exposed surface area of 3D graphene networks (Figures 12a and b), the core-sheath GF@3D-G offers great advantages as flexible electrodes for efficient fiber-based 
a
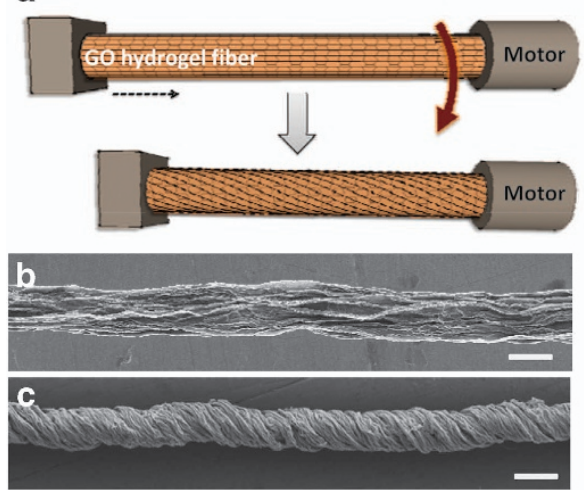

e

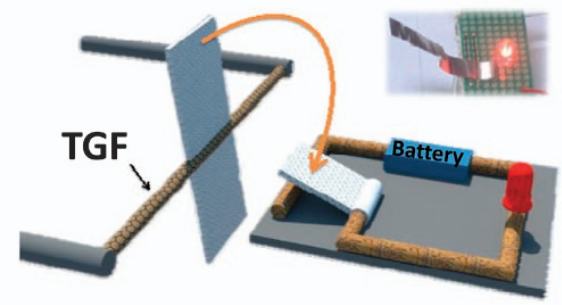

g

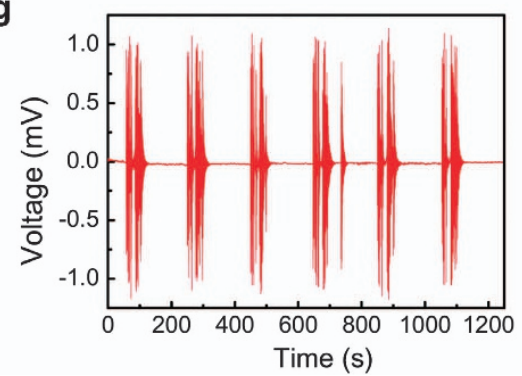

d

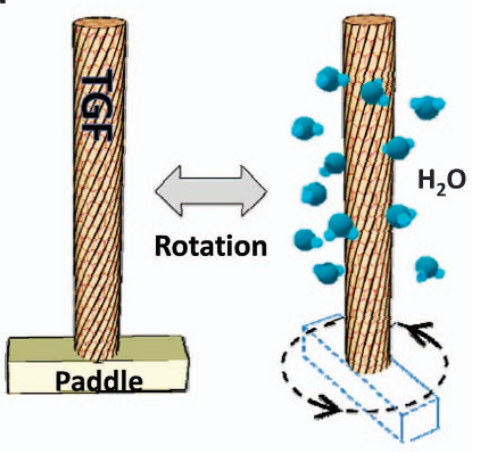

f

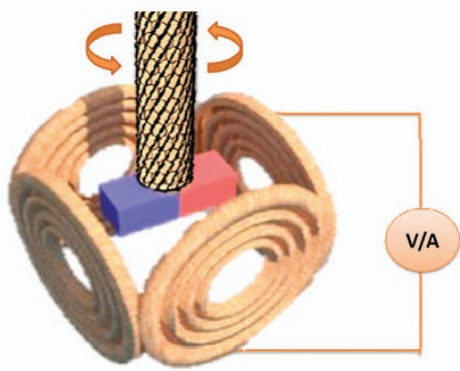

h

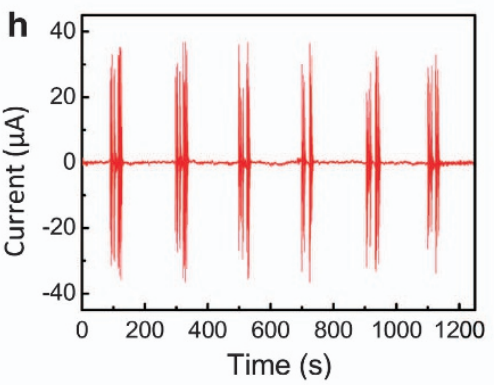

Figure 9 (a) Scheme of the twisted graphene fiber (TGF) fabrication. (b, c) Scanning electron microscope (SEM) images of the directly dried graphene oxide (GO) fiber and TGF formed with an applied 5000 turns per meter, respectively. Scale bars, $50 \mu \mathrm{m}$. (d) Schematic rotation of a TGF with a paddle at low (left) and high (right) humidity. When the moisture increases (right), the TGF can drive the paddle rotating fast; then the paddle can reverse to the initial state when the moisture decreases (left). (e, f) Scheme of the designed humidity switch and the alternating current generator based on the humidityresponsive TGFs. In the switch (e), TGF in response to moisture (for example, $\mathrm{RH}=85 \%$ ) can rotate a paddle to press on the metal plate, as indicated by the arrow, so that the electric circle powered by the battery will turn on the light emitting diode, as shown in the inset photo. The generator (f) contains four $\mathrm{Cu}$ coils around the TGF with a magnet. When the environment humidity changes, the TGF can reversibly rotate the magnet within the surrounding Cu coils to generate electricity. (g, h) Open-circuit voltage and short-circuit current of the generator tested under humidity changes between 20 and $85 \%$. Reproduced with permission. Copyright 2014 from Cheng et al. ${ }^{64}$

electrochemical supercapacitor. ${ }^{45}$ Fiber supercapacitors consist of two intertwined electrodes, both of which are solidified in $\mathrm{H}_{2} \mathrm{SO}_{4}$-PVA gel electrolyte for formation of an all solid-state fiber supercapacitor. The assembled fiber supercapacitor of GF@3D-G is highly flexible, which can be manipulated to a spring shape with highly compressible and stretchable properties, and can also be conveniently woven into textiles for wearable electronics (Figures $12 \mathrm{c}$ and d).

A novel hybrid fiber of $\mathrm{MnO}_{2}$-modified graphene sheets on GF has also been fabricated by direct deposition of $\mathrm{MnO}_{2}$ onto the graphene network surrounding the GF $\left(\mathrm{MnO}_{2} / \mathrm{G} / \mathrm{GF}\right){ }^{71}$ By combining the electric double layer capacitance of the graphene network with the pseudocapacitance of the $\mathrm{MnO}_{2}$ nanostructures, the all solidstate fiber supercapacitor shows greatly enhanced electrochemical capacitive behavior with robust tolerance to mechanical deformation. However, the graphene-CNT hybrid fibers can be further woven into textile electrodes for the construction of flexible supercapacitors with a high tolerance to repeated bending cycles. ${ }^{44}$ Various other applications, such as catalysis, separation and adsorption, can be envisioned for the graphene-CNT hybrid fibers. Other examples of flexible all solid-state supercapacitors based on GFs and assembled graphenes on fibers can be found in references. ${ }^{72-74}$

\section{Solid-phase microextraction (SPME) sorbents}

SPME has been widely used in the analysis of trace organic contaminants ${ }^{75}$ because of its simple operation and easy coupling to separation techniques, such as gas chromatography (GC), liquid chromatography, capillary electrophoresis and ion chromatography. ${ }^{76-78}$ When coupled with GC, SPME integrates sampling, isolation, enrichment and injection steps, making the analysis much more convenient. To date, several coating fibers are commercially available for SPME, including PDMS, polyacrylate, divinylbenzene, carboxen, carbowax and their copolymers. ${ }^{75,76}$ These commercial fibers generally exhibit some drawbacks, such as high cost, thermal, mechanical and/or chemical instability, insufficient 

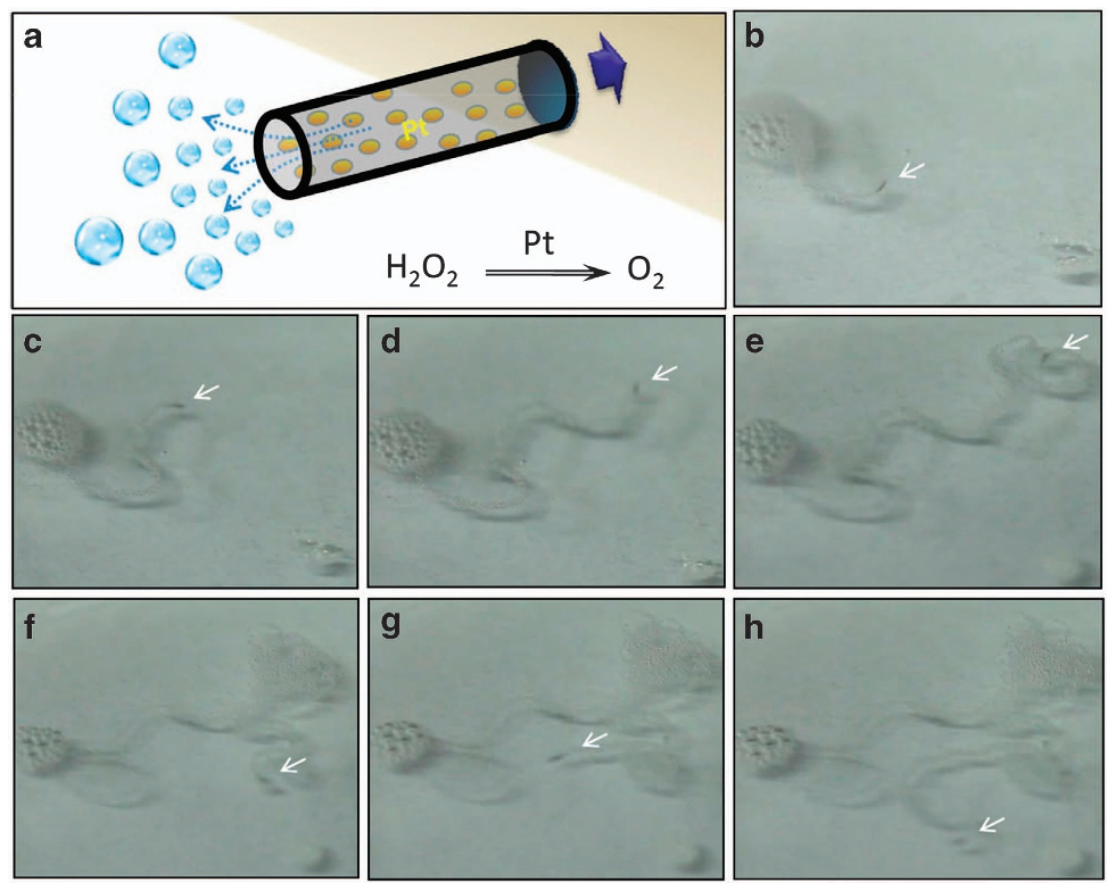

Figure 10 The hollow graphene fiber (hGF) micromotor. (a) Scheme of a moving micromotor. A one-end sealed hGF inner-wall modified with Pt nanoparticles propelled in an aqueous $\mathrm{H}_{2} \mathrm{O}_{2}$ solution. (b-h) Snapshots of the track for a moving hGF micromotor in a 20 wt\% $\mathrm{H}_{2} \mathrm{O}_{2}$ solution. Reproduced with permission. Copyright 2012 from Hu et al. ${ }^{25}$
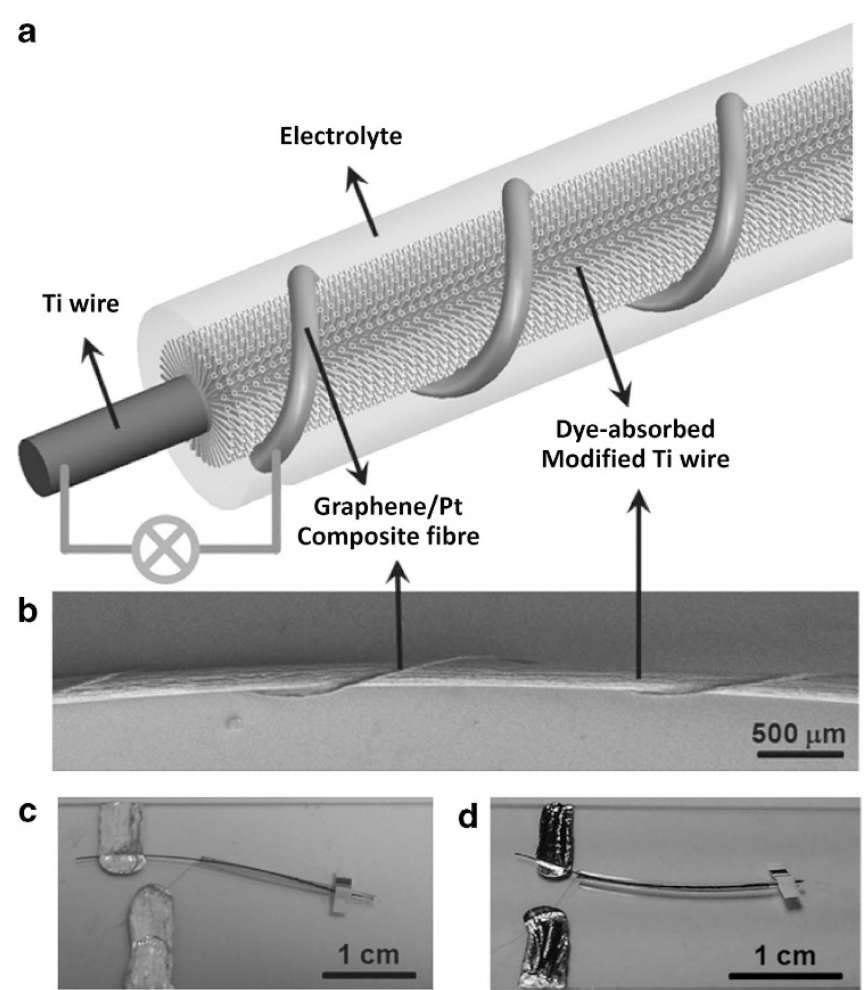

Figure 11 Dye-sensitized photovoltaic wire prepared by using a graphene/Pt composite fiber as the counter electrode and a Ti wire impregnated with $\mathrm{TiO}_{2}$ nanotubes as the working electrode. (a) Schematic illustration. (b) Scanning electron microscope (SEM) image. (c, d) Photographs of photovoltaic wires sealed in a capillary glass tube and flexible fluorinated ethylene propylene tube, respectively. Reproduced with permission. Copyright 2013 from Yang et al. ${ }^{68}$ selectivity, fragility of the fused-silica substrate, and limited range of polarity and reuse times, which largely restrict the application of SPME.

Graphene was first used as an SPME coating for extraction of pyrethroid pesticides from environmental water samples by Chen et al. ${ }^{79}$ Compared with commercial fibers, these graphene-based fibers show not only higher extraction efficiencies toward the targeted analytes but also far superior thermal and mechanical stabilities. Feng et al. ${ }^{80}$ used a hydrothermally synthesized GF for SPME, the performance of which was investigated through the analysis of five organochlorine pesticides using GC/electron capture detector (GC/ ECD). The results indicated that the GFs not only exhibited higher enrichment factors toward organochlorine pesticides compared with PDMS fibers but also possessed higher thermal stability and longer service life compared with commercial fibers. Finally, a headspace SPME (HS-SPME) technique for the determination of organochlorine pesticides in water samples was developed using GF as the sorbent coupled to GC/ECD (HS-SPME-GC/ECD). Similarly, the monolithic GFs coupled to capillary GC in a direct-immersion mode achieved higher extraction efficiencies for aromatics than those for n-alkanes, especially for polycyclic aromatic hydrocarbons as a result of $\pi-\pi$ stacking interactions and hydrophobic effects. ${ }^{81}$ In addition, the fibers exhibited excellent durability and can be repetitively used $>160$ times without significant loss of extraction performance.

\section{Other applications}

Aside from the applications mentioned above, graphene-based fibers could also be used in other interesting fields. For example, the electrospinning process can incorporate graphene sheets into a continuous nanoscale composite fiber, which could be applied as an optical element in fiber lasers. Loh et al. ${ }^{50}$ presented that electrospun graphene nanocomposites are promising candidates as saturable absorbers with wideband absorption, which could be used as 

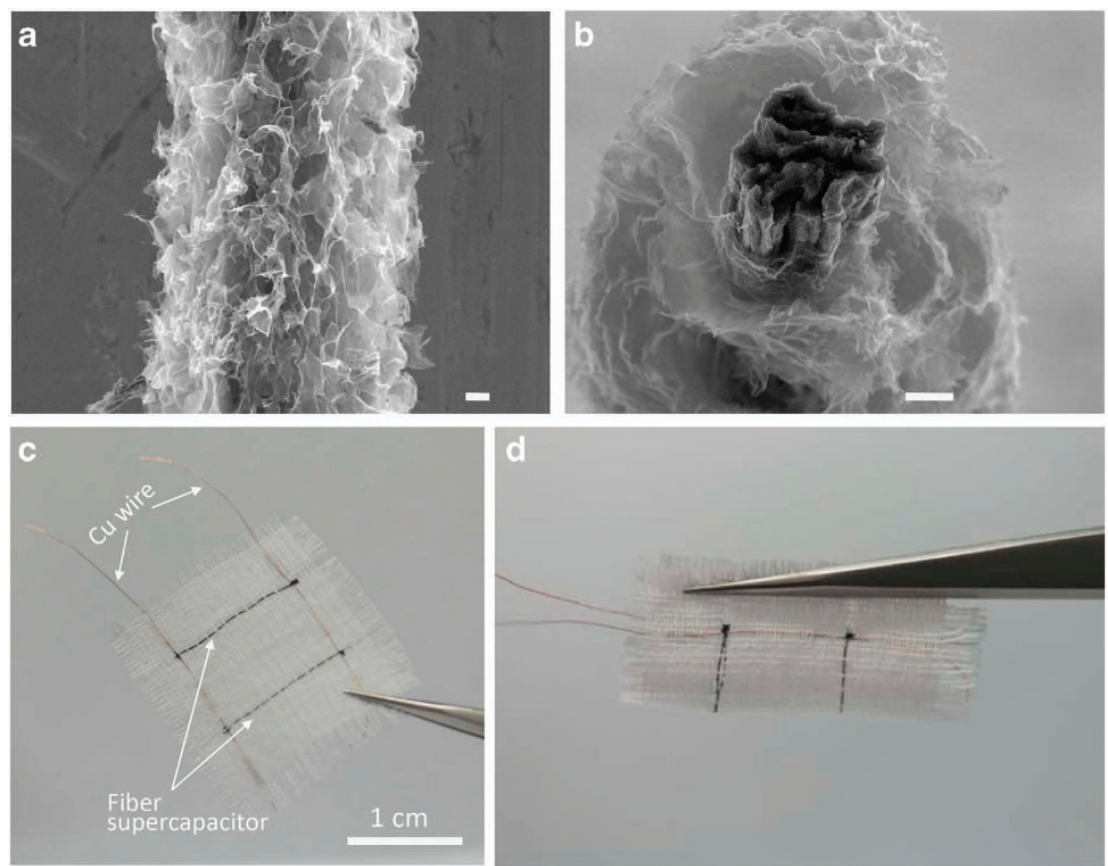

Figure 12 (a) Scanning electron microscope (SEM) images of a GF@3D-G and (b) cross-sectional view of a GF@3D-G showing the core graphene fiber (GF) surrounded by standing graphene sheets. Scale bars: $100 \mu \mathrm{m}$. (c, d) Photos of the textile embedded with two GF@3D-G fiber supercapacitors (each approximately $2 \mathrm{~cm}$ in length) in the flat and bending state, respectively. Reproduced with permission. Copyright 2013 from Meng et al. ${ }^{45}$

practical and efficient photonic materials for the generation of ultra short pulses in fiber lasers, attaining a larger modulation depth and smaller nonsaturable loss than SWNTs. By using graphene-polymer composites, it is possible to realize full waveband ultrafast lasers.

\section{SUMMARY AND OUTLOOK}

The active research in GFs during recent years has revealed numerous interesting findings and breakthroughs on the fabrication and applications of these fascinating materials. In particular, the integration of graphene nanosheets into macroscopic fibers provides a new material platform for developing unconventional smart systems and devices. Various preparation methods have been developed thus far for large scale production of GFs, which have paved the way for diverse applications of GFs in the future. Accordingly, many of the in situ and post-synthetic functional approaches and the available composites provide GFs with new functions and properties. Impressive examples for various applications include fiber-type actuators, robots, motors, photovoltaic cells and supercapacitors.

Compared with the long-term development of common carbon fibers, research on GFs is still in its early stages and there is no systematic theory established thus far to support this specific field. Many challenges still remain to be solved. For example, more effort needs to be continuously devoted to the high mechanical strength and electric conductivity of GFs. The highest strength achieved thus far is only $0.65 \mathrm{GPa}$ for biomimetic GFs. ${ }^{41}$ As a new type of fiber, GFs are still inferior to metal wires and high-performance carbon fibers in both mechanical and electrical properties, which mainly results from the relatively loosely stacked structure of graphene nanosheets within the fiber bodies. For high-strength fiber applications, the mechanical properties of GFs need further enhancement to reach values comparable to that of high quality carbon fibers. As a light-weight conductive wire, the electric conductivity of GFs is still low. The introduction of cross-linkers or metal nanowires into GFs for new hybrids $^{38,46}$ may be one method for solving this challenge. Furthermore, control over the preparation process of GFs requires deep study. GFs are mainly assembled from graphene or GO nanosheets with uncertain sizes, defects, shapes and chemical compositions. These factors strongly affect the assembly process and thus determine all the aspects of GF properties. For example, large and small graphene sizes can produce GFs with much different mechanical properties. ${ }^{21}$ However, most of the current studies mainly focus on the establishment of new strategies for GF fabrication, and the systematic consideration of all the factors for the final performance is generally absent. The precise modulation and control of the assembly of graphene sheets for GFs also remains a challenge. Although the production of GFs has been achieved through the continuous process of the wet-spinning method, the generation of practical products based on these unique GFs requires the cooperation of more researchers from various fields.

In summary, the macroscopic assembly of graphene into GFs has rendered new opportunities for the development of new materials and devices over common fibers. With the progress on the understanding and control of the assembly of graphene, high-performance GFs will be achieved in the near future and new applications will also be revealed beyond smart electronic textiles.

\section{CONFLICT OF INTEREST}

The authors declare no conflict of interest.

\section{ACKNOWLEDGEMENTS}

The authors thank the 973 program (2011CB013000) of China and NSFC (21325415, 21174019 and 51161120361), the Fok Ying Tong Education Foundation (no. 131043) and the 111 Project 807012 for their financial support. 
1 Dalton, A. B., Collins, S., Munoz, E., Razal, J. M., Ebron, V. H., Ferraris, J. P., Coleman, J. N., Kim, B. G. \& Baughman, R. H. Super-tough carbon-nanotube fibres-these extraordinary composite fibres can be woven into electronic textiles. Nature 423, 703-703 (2003)

2 Ericson, L. M., Fan, H., Peng, H. Q., Davis, V. A., Zhou, W., Sulpizio, J., Wang, Y. H., Booker, R., Vavro, J., Guthy, C., Parra-Vasquez, A. N. G., Kim, M. J., Ramesh, S. Saini, R. K., Kittrell, C., Lavin, G., Schmidt, H., Adams, W. W., Billups, W. E., Pasquali, M., Hwang, W. F., Hauge, R. H., Fischer, J. E. \& Smalley, R. E. Macroscopic neat, single-walled carbon nanotube fibers. Science 305, 1447-1450 (2004).

3 Vigolo, B., Penicaud, A., Coulon, C., Sauder, C., Pailler, R., Journet, C., Bernier, P. \& Poulin, P. Macroscopic fibers and ribbons of oriented carbon nanotubes. Science 290, 1331-1334 (2000)

4 Davis, V. A., Parra-Vasquez, A. N. G., Green, M. J., Rai, P. K., Behabtu, N., Prieto, V., Booker, R. D., Schmidt, J., Kesselman, E., Zhou, W., Fan, H., Adams, W. W., Hauge, R. H., Fischer, J. E., Cohen, Y., Talmon, Y., Smalley, R. E. \& Pasquali, M. True solutions of single-walled carbon nanotubes for assembly into macroscopic materials. Nat. Nanotechnol 4, 830-834 (2009)

5 Jiang, K. L., Li, Q. Q. \& Fan, S. S. Nanotechnology: spinning continuous carbon nanotube yarns-carbon nanotubes weave their way into a range of imaginative macroscopic applications. Nature 419, 801-801 (2002)

$6 \mathrm{Li}, \mathrm{Y}$. L., Kinloch, I. A. \& Windle, A. H. Direct spinning of carbon nanotube fibers from chemical vapor deposition synthesis. Science 304, 276-278 (2004)

7 Zhang, M., Atkinson, K. R. \& Baughman, R. H. Multifunctional carbon nanotube yarns by downsizing an ancient technology. Science 306, 1358-1361 (2004).

8 Zhang, X. B., Jiang, K. L., Teng, C., Liu, P., Zhang, L., Kong, J., Zhang, T. H., Li, Q. Q. \& Fan, S. S. Spinning and processing continuous yarns from 4-inch wafer scale superaligned carbon nanotube arrays. Adv. Mater. 18, 1505-1510 (2006)

9 Geim, A. K. \& Novoselov, K. S. The rise of graphene. Nat. Mater. 6, 183-191 (2006)

10 Novoselov, K. S., Geim, A. K., Morozov, S. V., Jiang, D., Zhang, Y., Dubonos, S. V. Grigorieva, I. V. \& Firsov, A. A. Electric field effect in atomically thin carbon films. Science 306, 666-669 (2004).

11 Novoselov, K. S., Geim, A. K., Morozov, S. V., Jiang, D., Katsnelson, M. I., Grigorieva, I. V., Dubonos, S. V. \& Firsov, A. A. Two-dimensional gas of massless Dirac fermions in graphene. Nature 438, 197-200 (2005).

12 Zhang, Y. B., Tan, Y. W., Stormer, H. L. \& Kim, P. Experimental observation of the quantum Hall effect and Berry's phase in graphene. Nature 438, 201-204 (2005).

13 Balandin, A. A., Ghosh, S., Bao, W. Z., Calizo, I., Teweldebrhan, D., Miao, F. \& Lau, C. N. Superior thermal conductivity of single-layer graphene. Nano Lett. 8, 902-907 (2008).

14 Lee, C., Wei, X. D., Kysar, J. W. \& Hone, J. Measurement of the elastic properties and intrinsic strength of monolayer graphene. Science 321, 385-388 (2008).

15 Xu, Y. X., Sheng, K. X., Li, C. \& Shi, G. Q. Self-assembled graphene hydrogel via a onestep hydrothermal process. ACS Nano 4, 4324-4330 (2010).

$16 \mathrm{Xu}, \mathrm{Z}$. \& Gao, C. Graphene chiral liquid crystals and macroscopic assembled fibres. Nat. Commun. 2, 571 (2011).

17 Xu, Z., Zhang, Y., Li, P. G. \& Gao, C. Strong, conductive, lightweight, neat graphene aerogel fibers with aligned pores. ACS Nano 6, 7103-7113 (2012).

18 Jalili, R., Aboutalebi, S. H., Esrafilzadeh, D., Shepherd, R. L., Chen, J., Aminorroaya-Yamini, S., Konstantinov, K., Minett, A. I., Razal, J. M. \& Wallace, G. G. Scalable one-step wet-spinning of graphene fibers and yarns from liquid crystalline dispersions of graphene oxide: towards multifunctional textiles. Adv. Funct. Mater. 23, 5345-5354 (2013).

19 Cong, H. P., Ren, X. C., Wang, P. \& Yu, S. H. Wet-spinning assembly of continuous, neat, and macroscopic graphene fibers. Sci. Rep. 2, 613 (2012).

20 Chen, L., He, Y. L., Chai, S. G., Qiang, H., Chen, F. \& Fu, Q. Toward high performance graphene fibers. Nanoscale 5, 5809-5815 (2013).

21 Xiang, C. S., Young, C. C., Wang, X., Yan, Z., Hwang, C. C., Cerioti, G., Lin, J., Kono, J., Pasquali, M. \& Tour, J. M. Large flake graphene oxide fibers with unconventional $100 \%$ knot efficiency and highly aligned small flake graphene oxide fibers. Adv. Mater. 25 4592-4597 (2013)

22 Zhao, Y., Jiang, C. C., Hu, C. G., Dong, Z. L., Xue, J. L., Meng, Y. N., Zheng, N. Chen, P. W. \& Qu, L. T. Large-scale spinning assembly of neat, morphology-defined graphene-based hollow fibers. ACS Nano 7, 2406-2412 (2013).

23 Sun, J. K., Li, Y. H., Peng, Q. Y., Hou, S. C., Zou, D. C., Shang, Y. Y., Li, Y. B., Li, P. X. Du, Q. J., Wang, Z. H., Xia, Y. Z., Xia, L. H., Li, X. L. \& Cao, A. Y. Macroscopic, flexible, high-performance graphene ribbons. ACS Nano 7, 10225-10232 (2013).

24 Dong, Z. L., Jiang, C. C., Cheng, H. H., Zhao, Y., Shi, G. Q., Jiang, L. \& Qu, L. T. Facile fabrication of light, flexible and multifunctional graphene fibers. Adv. Mater. 24, 1856-1861 (2012)

25 Hu, C. G., Zhao, Y., Cheng, H. H., Wang, Y. H., Dong, Z. L., Jiang, C. C., Zhai, X. Q., Jiang, L. \& Qu, L. T. Graphene microtubings: controlled fabrication and site-specific functionalization. Nano Lett. 12, 5879-5884 (2012).

26 Li, X. M., Zhao, T. S., Wang, K. L., Yang, Y., Wei, J. Q., Kang, F. Y., Wu, D. H. \& Zhu, H. W. Directly drawing self-assembled, porous, and monolithic graphene fiber from chemical vapor deposition grown graphene film and its electrochemical properties. Langmuir. 27, 12164-12171 (2011).

27 Li, X., Sun, P. Z., Fan, L. L., Zhu, M., Wang, K. L., Zhong, M. L., Wei, J. Q., Wu, D. H. Cheng, Y. \& Zhu, H. W. Multifunctional graphene woven fabrics. Sci. Rep. 2, 395 (2012).

28 Hu, C. G., Zhai, X. Q., Liu, L. L., Zhao, Y., Jiang, L. \& Qu, L. T. Spontaneous reduction and assembly of graphene oxide into three-dimensional graphene network on arbitrary conductive substrates. Sci. Rep. 3, 2065 (2013).
29 Kosynkin, D. V., Higginbotham, A. L., Sinitskii, A., Lomeda, J. R., Dimiev, A. Price, B. K. \& Tour, J. M. Longitudinal unzipping of carbon nanotubes to form graphene. Nature 458, 872-876 (2009).

30 Jiao, L. Y., Zhang, L., Wang, X. R., Diankov, G. \& Dai, H. J. Narrow graphene nanoribbons from carbon nanotubes. Nature 458, 877-880 (2009).

31 Cano-Marquez, A. G., Rodriguez-Macias, F. J., Campos-Delgado, J., Espinosa-Gonzalez, C. G., Tristan-Lopez, F., Ramirez-Gonzalez, D., Cullen, D. A., Smith, D. J., Terrones, M. \& Vega-Cantu, Y. I. Ex-MWNTs: graphene sheets and ribbons produced by lithium intercalation and exfoliation of carbon nanotubes. Nano Lett. 9, 1527-1533 (2009).

32 Carretero-Gonzalez, J., Castillo-Martinez, E., Dias-Lima, M., Acik, M., Rogers, D. M., Sovich, J., Haines, C. S., Lepro, X., Kozlov, M., Zhakidov, A., Chabal, Y. \& Baughman, R. H. Oriented graphene nanoribbon yarn and sheet from aligned multiwalled carbon nanotube sheets. Adv. Mater. 24, 5695-5701 (2012).

33 Xiang, C. S., Behabtu, N., Liu, Y. D., Chae, H. G., Young, C. C., Genorio, B., Tsentalovich, D. E., Zhang, C. G., Kosynkin, D. V., Lomeda, J. R., Hwang, C. C., Kumar, S., Pasquali, M. \& Tour, J. M. Graphene nanoribbons as an advanced precursor for making carbon fiber. ACS Nano 7, 1628-1637 (2013).

34 Jang, E. Y., Carretero-Gonzalez, J., Choi, A., Kim, W. J., Kozlov, M. E., Kim, T., Kang, T. J., Baek, S. J., Kim, D. W., Park, Y. W., Baughman, R. H. \& Kim, Y. H. Fibers of reduced graphene oxide nanoribbons. Nanotechnology 23, 235601 (2012).

35 Tian, Z. S., Xu, C. X., Li, J. T., Zhu, G. Y., Shi, Z. L. \& Lin, Y. Self-assembled freestanding graphene oxide fibers. ACS Appl. Mater. Interfaces 5, 1489-1493 (2013).

36 Zhao, Y., Song, L., Zhang, Z. P. \& Qu, L. T. Stimulus-responsive graphene systems towards actuator applications. Energy Environ. Sci. 6, 3520-3536 (2013).

37 Zhang, J., Zhang, Z. P., Chen, N. \& Qu, L. T. Environmentally responsive graphene systems. Small. 10, 2151-2164 (2014).

38 Xu, Z., Liu, Z., Sun, H. Y. \& Gao, C. Highly electrically conductive Ag-doped graphene fibers as stretchable conductors. Adv. Mater. 25, 3249-3253 (2013).

$39 \mathrm{Xu}$, Z., Sun, H. Y., Zhao, X. L. \& Gao, C. Ultrastrong fibers assembled from giant graphene oxide sheets. Adv. Mater. 25, 188-193 (2013)

$40 \mathrm{Hu}$, X. Z., Xu, Z. \& Gao, C. Multifunctional, supramolecular, continuous artificial nacre fibres. Sci. Rep. 2, 767 (2012).

$41 \mathrm{Hu}$, X. Z., Xu, Z., Liu, Z. \& Gao, C. Liquid crystal self-templating approach to ultrastrong and tough biomimic composites. Sci. Rep. 3, 2374 (2013).

42 Zhao, X. L., Xu, Z., Zheng, B. N. \& Gao, C. Macroscopic assembled, ultrastrong and $\mathrm{H}_{2} \mathrm{SO}_{4}$-resistant fibres of polymer-grafted graphene oxide. Sci. Rep. 3, 3164 (2013).

43 Zhong, X. H., Wang, R., Wen, Y. Y. \& Li, Y. L. Carbon nanotube and graphene multiplethread yarns. Nanoscale 5, 1183-1187 (2013).

44 Cheng, H. H., Dong, Z. L., Hu, C. G., Zhao, Y., Hu, Y., Qu, L. T., Che, N. \& Dai, L. M. Textile electrodes woven by carbon nanotube-graphene hybrid fibers for flexible electrochemical capacitors. Nanoscale 5, 3428-3434 (2013).

45 Meng, Y. N., Zhao, Y., Hu, C. G., Cheng, H. H., Hu, Y., Zhang, Z. P., Shi, G. Q. \& Qu, L. T. All-graphene core-sheath microfibers for all-solid-state, stretchable fibriform supercapacitors and wearable electronic textiles. Adv. Mater. 25, 2326-2331 (2013)

46 Shin, M. K., Lee, B., Kim, S. H., Lee, J. A., Spinks, G. M., Gambhir, S., Wallace, G. G. Kozlov, M. E., Baughman, R. H. \& Kim, S. J. Synergistic toughening of composite fibres by self-alignment of reduced graphene oxide and carbon nanotubes. Nat. Commun. 3, 650 (2012).

47 Matsumoto, H., Imaizumi, S., Konosu, Y., Ashizawa, M., Minagawa, M., Tanioka, A., Lu, W. \& Tour, J. M. Electrospun composite nanofiber yarns containing oriented graphene nanoribbons. ACS Appl. Mater. Interfaces 5, 6225-6231 (2013).

48 Qi, Y. Y., Tai, Z. X., Sun, D. F., Chen, J. T., Ma, H. B., Yan, X. B., Liu, B. \& Xue, Q. J. Fabrication and characterization of poly(vinyl alcohol)/graphene oxide nanofibrous biocomposite scaffolds. J. Appl. Polym. Sci. 127, 1885-1894 (2013).

49 Wang, C., Li, Y. D., Ding, G. Q., Xie, X. M. \& Jiang, M. H. Preparation and characterization of graphene oxide/poly(vinyl alcohol) composite nanofibers via electrospinning. J. Appl. Polym. Sci. 127, 3026-3032 (2013).

50 Bao, Q. L., Zhang, H., Yang, J. X., Wang, S., Tong, D. Y., Jose, R., Ramakrishna, S., Lim, C. T. \& Loh, K. P. Graphene-polymer nanofiber membrane for ultrafast photonics. Adv. Funct. Mater. 20, 782-791 (2010).

51 Jiang, Z. X., Li, Q., Chen, M. L., Li, J. B., Li, J., Huang, Y. D., Besenbacher, F. \& Dong, M. D. Mechanical reinforcement fibers produced by gel-spinning of poly-acrylic acid (PAA) and graphene oxide (GO) composites. Nanoscale 5, 6265-6269 (2013).

52 Huang, Y., Liang, J. J. \& Chen, Y. S. The application of graphene based materials for actuators. J. Mater. Chem. 22, 3671-3679 (2012)

53 Zhu, C. H., Lu, Y., Peng, J., Chen, J. F. \& Yu, S. H. Photothermally sensitive poly(N-isopropylacrylamide)/graphene oxide nanocomposite hydrogels as remote lightcontrolled liquid microvalves. Adv. Funct. Mater. 22, 4017-4022 (2012).

54 Wu, C. Z., Feng, J., Peng, L. L., Ni, Y., Liang, H. Y., He, L. H. \& Xie, Y. Large-area graphene realizing ultrasensitive photothermal actuator with high transparency: new prototype robotic motions under infrared-light stimuli. J. Mater. Chem. 21, 18584-18591 (2011)

55 Zhang, J., Zhao, F., Zhang, Z. P., Chen, N. \& Qu, L. T. Dimension-tailored functional graphene structures for energy conversion and storage. Nanoscale 5, 3112-3126 (2013)

56 Lu, L. H., Liu, J. H., Hu, Y., Zhang, Y. W. \& Chen, W. Graphene-stabilized silver nanoparticle electrochemical electrode for actuator design. Adv. Mater. 25, 1270-1274 (2013).

57 Liang, J. J., Huang, L., Li, N., Huang, Y., Wu, Y. P., Fang, S. L., Oh, J. Y., Kozlov, M., Ma, Y. F., Li, F. F., Baughman, R. \& Chen, Y. S. Electromechanical actuator with controllable motion, fast response rate, and high-frequency resonance based on graphene and polydiacetylene. ACS Nano 6, 4508-4509 (2012). 
58 Xie, X. J., Qu, L. T., Zhou, C., Li, Y., Zhu, J., Bai, H., Shi, G. Q. \& Dai, L. M. An Asymmetrically surface-modified graphene film electrochemical actuator. ACS Nano 4, 6050-6054 (2010).

59 Xie, X. J., Bai, H., Shi, G. Q. \& Qu, L. T. Load-tolerant, highly strain-responsive graphene sheets. J. Mater. Chem. 21, 2057-2059 (2011).

60 Liu, J., Wang, Z., Xie, X. J., Cheng, H. H., Zhao, Y. \& Qu, L. T. A rationally-designed synergetic polypyrrole/graphene bilayer actuator. J. Mater. Chem. 22, 4015-4020 (2012).

61 Liu, J., Wang, Z., Zhao, Y., Cheng, H. H., Hu, C. A., Jiang, L. \& Qu, L. T. Three-dimensional graphene-polypyrrole hybrid electrochemical actuator. Nanoscale 4, 7563-7568 (2012)

62 Wang, Y. H., Bian, K., Hu, C. G., Zhang, Z. P., Chen, N., Zhang, H. M. \& Qu, L. T. Flexible and wearable graphene/polypyrrole fibers towards multifunctional actuator applications. Electrochem. Commun. 35, 49-52 (2013).

63 Cheng, H. H., Liu, J., Zhao, Y., Hu, C. G., Zhang, Z. P., Chen, N., Jiang, L. \& Qu, L. T. Graphene fibers with predetermined deformation as moisture-triggered actuators and robots. Angew. Chem. Int. Ed. 52, 10482-10486 (2013).

64 Cheng, H. H., Hu, Y., Zhao, F., Dong, Z. L., Wang, Y. H., Chen, N., Zhang, Z. P. \& Qu, L. T. Moisture-activated torsional graphene-fiber motor. Adv. Mater. 26, 2909-2913 (2014).

65 Paxton, W. F., Sundararajan, S., Mallouk, T. E. \& Sen, A. Chemical locomotion. Angew. Chem., Int. Ed. 45, 5420-5429 (2006).

66 Sanchez, S. \& Pumera, M. Nanorobots: the ultimate wireless self-propelled sensing and actuating devices. Chem. Asian J. 4, 1402-1410 (2009).

67 Mirkovic, T., Zacharia, N. S., Scholes, G. D. \& Ozin, G. A. Fuel for thought: chemically powered nanomotors out-swim nature's flagellated bacteria. ACS Nano 4, 1782-1789 (2010).

68 Yang, Z. B., Sun, H., Chen, T., Qiu, L. B., Luo, Y. F. \& Peng, H. S. Photovoltaic wire derived from a graphene composite fiber achieving an $8.45 \%$ energy conversion efficiency. Angew. Chem. Int. Ed. 52, 7545-7548 (2013).

69 Chen, J., Li, C. \& Shi, G. Graphene materials for electrochemical capacitors. J. Phys. Chem. Lett 4, 1244-1253 (2013).

70 Huang, L., Li, C. \& Shi, G. High-performance and flexible electrochemical capacitors based on graphene/polymer composite films. J. Mater. Chem. A 2, 968-974 (2014).

71 Chen, Q., Meng, Y. N., Hu, C. G., Zhao, Y., Shao, H. B., Chen, N. \& Qu, L. T. $\mathrm{MnO}_{2}$-modified hierarchical graphene fiber electrochemical supercapacitor. J. Power Sources 247, 32-39 (2014).

72 Li, X. M., Zhao, T. S., Chen, Q., Li, P. X., Wang, K. L., Zhong, M. L., Wei, J. Q., Wu, D. H., Wei, B. Q. \& Zhu, H. W. Flexible all solid-state supercapacitors based on chemical vapor deposition derived graphene fibers. Phys. Chem. Chem. Phys. 15, 17752-17757 (2013).

73 Huang, T. Q., Zheng, B. N., Kou, L., Gopalsamy, K., Xu, Z., Gao, C., Meng, Y. N. \& Wei, Z. X. Flexible high performance wet-spun graphene fiber supercapacitors. RSC Adv 3, 23957-23962 (2013).

74 Li, Y., Sheng, K., Yuan, W. \& Shi, G. A high-performance flexible fibre-shaped electrochemical capacitor based on electrochemically reduced graphene oxide. Chem. Commun. 49, 291-293 (2013).

75 Dietz, C., Sanz, J. \& Camara, C. Recent developments in solid-phase microextraction coatings and related techniques. J. Chromatogr. A 1103, 183-192 (2006).

76 Spietelun, A., Pilarczyk, M., Kloskowski, A. \& Namiesnik, J. Current trends in solidphase microextraction (SPME) fibre coatings. Chem. Soc. Rev. 39, 4524-4537 (2010).

77 Kaykhaii, M., Dicinoski, G. W., Smedley, R., Pawliszyn, J. \& Haddad, P. R. Preparation and evaluation of solid-phase microextraction fibres based on functionalized latex nanoparticle coatings for trace analysis of inorganic anions. J. Chromatogr. A 1217, 3452-3456 (2010).

78 Li, Q. L., Ding, Y. J. \& Yuan, D. X. Electrosorption-enhanced solid-phase microextraction of trace anions using a platinum plate coated with single-walled carbon nanotubes. Talanta 85, 1148-1153 (2011).

79 Chen, J. M., Zou, J., Zeng, J. B., Song, X. H., Ji, J. J., Wang, Y. R., Ha, J. \& Chen, X. Preparation and evaluation of graphene-coated solid-phase microextraction fiber. Anal. Chim. Acta 678, 44-49 (2010).

80 Luo, Y. B., Yuan, B. F., Yu, Q. W. \& Feng, Y. Q. Substrateless graphene fiber: a sorbent for solid-phase microextraction. J. Chromatogr. A 1268, 9-15 (2012).

81 Fan, J., Dong, Z. L., Qi, M. L., Fu, R. N. \& Qu, L. T. Monolithic graphene fibers for solid-phase microextraction. J. Chromatogr. A 1320, 27-32 (2013).

(i) (5) $\Theta$ This work is licensed under a Creative Commons Attribution-NonCommercial-NoDerivs 3.0 Unported License. The images or other third party material in this article are included in the article's Creative Commons license, unless indicated otherwise in the credit line; if the material is not included under the Creative Commons license, users will need to obtain permission from the license holder to reproduce the material. To view a copy of this license, visit http://creativecommons.org/licenses/by-nc-nd/3.0/
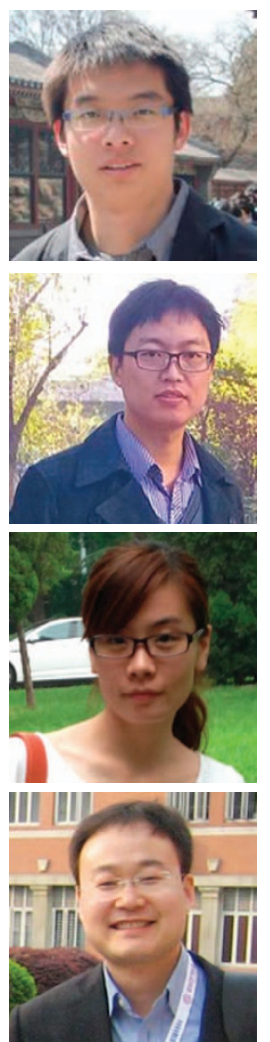

Huhu Cheng is currently a PhD candidate under the supervision of Professor Liangti Qu at the School of Chemistry in Beijing Institute of Technology. His research focuses on assembly of graphene-based materials for smart devices.

Chuangang Hu is currently a $\mathrm{PhD}$ student in the research group of functional nanostructures supervised by Professor Liangti $\mathrm{Qu}$ at Beijing Institute of Technology. His research focus is the functionalization of graphenes for energy conversion and storage applications.

Yang Zhao is currently a PhD student under the supervision of Professor Liangti Qu at the School of Chemistry in Beijing Institute of Technology. Her research interests lie in the development of new methods and strategies for fabrication of carbon-based functional materials for various applications.

Liangti Qu received a $\mathrm{PhD}$ in chemistry from Tsinghua University (Beijing, China) in 2004. He becomes a professor of chemistry at Beijing Institute of Technology in 2009 after working at University of Dayton in Ohio, USA for 5 years. As Xu-Teli Distinguished Professor, he now leads the Nanocarbon research group with interest in material chemistry mainly focusing on the synthesis, functionalization and application of nanomaterials with carbon-carbon conjugated structures including graphenes, carbon nanotubes and conducting polymers. So far, Dr Qu has published >110 scientific papers in the prestigious journals. 